\title{
Venture Capital Finance: A Security Design Approach
}

\author{
Rafael Repullo \\ CEMFI and CEPR
}

\author{
Javier Suarez \\ CEMFI and CEPR
}

November 2003

\begin{abstract}
This paper characterizes the optimal securities for venture capital finance in an environment with multiple investment stages and double-sided moral hazard in the relationship between entrepreneurs and venture capitalists. We show that if the conditions relevant for continuation into later stages are verifiable, the optimal security gives the venture capitalist a constant share in the success return of the project over a predetermined set of continuation states. Otherwise, the parties sign an initial start-up contract that is later renegotiated. In this case, in order to minimize the incentive distortions associated with the burden of early financing stages, the optimal start-up security gives a zero payoff in low profitability states and thereafter an increasing share in the success return of the project. (JEL: D92, G24, G32).
\end{abstract}

We would like to thank Tom Berglund, Sudipto Bhattacharya, Patrick Bolton, Paul Gompers, Antoine Faure-Grimaud, Jorge Padilla, Enrique Sentana, Jean Tirole, Rob Townsend, Masako Ueda, and especially Marco Pagano (the editor) for helpful comments. We also thank seminar participants at Austin, Berkeley, Berlin, Boston, Chicago, Frankfurt, Lisbon, London, Minnesota, New York, Penn, Stanford, and Toulouse. Financial support from the European Bank for Reconstruction and Development (EBRD) and the Spanish Ministry of Education (Grant No. PB98-0049) is gratefully acknowledged. Address for correspondence: CEMFI, Casado del Alisal 5, 28014 Madrid, Spain. Tel: 34-914290551. Fax: 34-914291056. E-mail: repullo@cemfi.es, suarez@cemfi.es. 


\section{Introduction}

Venture capital (VC) plays an important role in the financing of young firms seeking to grow rapidly. This mode of financing is characterized by three salient features. First, venture capitalists intervene very actively in the management of the firms that they fund: they use their experience, contacts, and reputation in order to provide advice to the entrepreneurs, especially with regard to issues such as the selection of qualified personnel or the dealing with suppliers and customers. ${ }^{1}$ Second, the infusion of capital occurs in stages, matching investment decisions based on information that arrives over time. ${ }^{2}$ Third, it relies on equity-like and convertible securities instead of the senior secured debt that characterizes most bank finance. ${ }^{3}$

This paper demonstrates that optimal contracts in a world with moral hazard are consistent with all three of these features. Instead of comparing VC finance under various combinations of standard securities, we characterize the securities that result from the solution to an optimal contract problem. We identify various setups where the payoff structure of these optimal securities resemble the equity-like and the convertible-like payoffs that are so frequently observed in $\mathrm{VC}$ finance. In order to keep the security design problem analytically tractable, we make a number of simplifying assumptions, and as a result the correspondence between the optimal securities that we derive and the empirically observed securities is not exact. Yet, as we further discuss below, the insights from this analysis help understand some of the specificities of $\mathrm{VC}$ contracting and suggest several avenues for a deeper empirical analysis of the staging features of VC finance. ${ }^{4}$

Formally, we analyze the financing problem of a new venture that faces (i) a sequence of investment stages (specifically, a start-up stage and an expansion stage) and (ii) a double-sided moral hazard problem concerning the managerial contributions of the entrepreneur and her venture capitalist. We study how the incentive problems that arise in the expansion stage influence the optimal payoff structure of

\footnotetext{
${ }^{1}$ See, for example, Gorman and Sahlman (1989), Sahlman (1990), and Kaplan and Strömberg (2001).

${ }^{2}$ The staging of finance has been documented, among others, by Gompers (1995).

${ }^{3}$ See, for example, Sahlman (1990) and Gompers (1997).

${ }^{4}$ Kaplan and Strömberg (2003) provide an interesting assessment of how the different theories on VC finance, including ours, match the facts, but their analysis barely deals with the staging aspects that we emphasize.
} 
the financial claims issued when the entrepreneur starts her contractual relationship with the venture capitalist.

Consider, for concreteness, the example of a biotech start-up. A scientist has an idea about how to obtain a new drug, but no money to develop it. The uncertainty on the applicability of the drug is high and it is unclear whether the scientist will be finally able to standardize the synthesizing process, to patent it before some competitor does, to get the approval of the health authorities, and to successfully commercialize the drug. Some start-up investment is needed to carry out the experiments that will clarify the applicability, potential market size, and expected profitability of the drug. If the prospects are sufficiently promising, some larger expansion investment will have to be undertaken. At that stage, the joint efforts of the scientist and some venture capitalist with significant managerial expertise in this type of venture will be essential for its final success. The questions to be addressed are how will the funding for each of the investment stages be provided and how will the future value of the venture be distributed between the entrepreneur and her venture capitalist.

We work out the optimal solution to the financing problem of an entrepreneur such as the scientist in the example. Such a problem is constrained by the characteristics of the environment that we consider, in particular the information available to the parties. ${ }^{5}$ A first characteristic is the unobservability of the efforts that the entrepreneur and the venture capitalist have to contribute during the expansion stage. This creates a double-sided moral hazard problem and, as we show, a complementarity between the provision of finance and advice, which makes it optimal to have a single venture capitalist as the firm's financier and advisor.

A second important characteristic is the verifiability of the information on potential profitability that arrives between the start-up stage and the expansion stage. When such interim information is verifiable, the entrepreneur and the venture capitalist can sign a contingent financing contract that establishes the terms for both the funding of the start-up investment and, contingent on the interim information on profitability, the funding of the expansion investment. In contrast, when the interim information on profitability is not verifiable, it is not possible to directly write the

\footnotetext{
${ }^{5}$ Notice that no revenue is generated during the development of the project and the investment is mostly in intangible assets, with small liquidation value. Hence bank-like financial arrangements based on liquidation threats (Hart and Moore, 1998; Repullo and Suarez, 1998) are not feasible.
} 
conditions for the continuation of the project into the initial contract, which is hence incomplete and potentially subject to renegotiation. ${ }^{6}$ In this context, we examine the empirically relevant case of stage financing whereby the funds for the start-up and the expansion investments are raised as they are needed.

We find that in both scenarios the double-sided moral hazard problem has a strong influence on the structure of the optimal securities. Since the entrepreneur's and the venture capitalist's incentives at the expansion stage improve with the potential profitability of the project, it is optimal to concentrate the compensation to the venture capitalist in the highest profitability states. In the case of contingent financing, this is achieved by directly allocating to him a constant final share in the success return of the project over a predetermined set of continuation states.

Under stage financing this arrangement would, of course, be equally desirable ex ante, but in general it violates the ex-post participation constraint of the venture capitalist. The parties sign an initial contract for the funding of the start-up investment only and, when the expansion investment has to be funded, this contract is renegotiated and a new contract is put in place. Because of its influence on the new contract, the payoff structure of the initial contract determines the distribution of the burden of the initial investment across continuation states. It turns out that, since in low profitability states funding the expansion investment will already require a large distortion in the venture capitalist's share, the optimal initial contract avoids further distortions by giving him no share in the project's success return in those states. As a counterpart of this, above some threshold, the initial contract gives the venture capitalist an increasing share in the success return. So the payoff structure of the optimal initial claim exhibits the non-linearity typical of a set of warrants that only yield a positive payoff if firm value exceeds the lowest strike price and pay more and more as firm value hits higher and higher strike prices.

The interactions between securities and incentives are central to the theoretical literature on VC finance. Various papers analyze the performance of different combinations of debt, equity, and convertibles in relation to the entrepreneurs' incentives on issues such as window dressing (Cornelli and Yosha, 2003) or fund diversion (Bergemann and Hege, 1998; Trester, 1998). Others focus on situations where the interests

\footnotetext{
${ }^{6}$ For an overview of the applications of the incomplete contracts paradigm to financial contracting, see Hart (2001).
} 
of entrepreneurs and venture capitalists are in conflict, and study how the securities held by the latter shape their incentives to exercise their control rights on investment (Admati and Pfleiderer, 1994), managerial replacement (Berglof, 1994), or liquidation (Marx, 1998).

Our paper is more closely related to yet another stream of the literature that has focused on the incentives concerning the managerial contributions of both entrepreneurs and venture capitalists. Casamatta (2003) assesses the performance of debt, equity, and convertibles in a model in which the efforts of the entrepreneur and the venture capitalist are substitutes. Schmidt (2003) shows that, if the entrepreneur and the venture capitalist contribute their (observable but not verifiable) effort sequentially, the use of convertibles allows to impose a credible punishment on the entrepreneur who deviates from the first best level of effort. Inderst and Müller (2003) introduce the double-sided moral hazard problem in an equilibrium search environment, and discuss how the effective level of competition in the market for venture capital alters the form of the equilibrium contracts. However none of these papers considers a multi-stage investment process and the related issue of how the verifiable or not verifiable nature of the information that arrives between stages affects the security design problem.

The novel aspects of our approximation shed light on a number of empirically relevant questions. Specifically, when objective performance indicators or "milestones" that are sufficiently correlated with the relevant future profitability variables can be found, we predict the use of straight equity and continuation terms explicitly contingent on the attainment of these milestones. This condition is more likely to hold in relatively more mature industries where some sort of explicit benchmarking is feasible, based on either past experience or the parallel performance of similar companies. When no explicit benchmarking is feasible, our analysis predicts that the start-ups will be more frequently discontinued before the expansion stage, and that the initial claims of the venture capitalists' will have a more complex payoff structure. In particular, they should allocate no share of the success returns to the venture capitalist below some threshold and, then, a larger and larger share of them as they increase, a structure that may be implemented by a combination of standard non-linear claims. These predictions are broadly consistent with the use of securities such as convert- 
ible preferred stock whose payoffs are concentrated in the highest profitability states. However, a formal test of our key implications would require an explicit analysis of the dynamics of VC stakes across financing rounds and their dependence on the venture's final performance.

The remaining sections are organized as follows. Section 2 presents the model. In Section 3 we analyze the financing of the expansion stage. Sections 4 and 5 characterize the optimal start-up contract under, respectively, verifiable and not verifiable interim information. In Section 6 we discuss several extensions such as the introduction of entrepreneurial wealth, relationship-specific information, and a general distribution of the bargaining power in interim renegotiations. Concluding remarks are in Section 7. Appendix A contains a general proof of the optimality of having a venture capitalist as the sole financier of the expansion stage. The proofs of the other results appear in Appendix B.

\section{The Model}

This section describes the ingredients of our model of venture capital finance. We first introduce the agents, technology, structure of information, and contracting possibilities. Then we describe the strategy for the analysis.

Agents and technology An entrepreneur has a project that requires an investment $I_{0}$ at an early start-up stage and an investment $I_{1}$ at a later expansion stage. If both investments are completed, the project reaches a cash-out stage in which it yields a random return $x$. This can be interpreted as the proceeds from either an IPO or the sale of the project to an established company. Otherwise, the project yields no return.

The project consists of a business opportunity (product, design or patent) whose potential profitability $\theta$ is uncertain until the completion of the start-up stage. This profitability translates into the final return $x$ only if the project is successfully developed during the expansion stage, which requires the effort of the entrepreneur, $e \in[0,1]$, and the advice of a venture capitalist, $a \in[0,1]$. The probability distribu- 
tion of the final return is

$$
x= \begin{cases}\theta, & \text { with probability } p(e, a), \\ 0, & \text { with probability } 1-p(e, a),\end{cases}
$$

where

$$
p(e, a)=e^{1-\alpha} a^{\alpha},
$$

with $\alpha \in(0,1)$. The Cobb-Douglas specification of the probability of success captures the complementarity between the effort provided by the entrepreneur (who has the original idea and some exclusive knowledge to develop it) and the advice provided by the venture capitalist (who has some previously accumulated business expertise, contacts and reputation that are relevant at this stage). The parameter $\alpha$ measures the relative productivity of the venture capitalist's advising effort.

The entrepreneur cares about the maximization of the expected value of her final wealth net of the disutility of her effort, $e$, which is

$$
U(e)=\frac{e^{2}}{2 u},
$$

where $u>0$. The entrepreneur has no initial wealth, so she requires external finance for the investments $I_{0}$ and $I_{1}$. This funding, as well as advice, can be provided by a large number of venture capitalists. Each of them maximizes the expected value of his final wealth net of the disutility of his advising effort, $a$, which is

$$
V(a)=\frac{a^{2}}{2 v},
$$

where $v>0$. Moreover, the opportunity cost of their funds is normalized to zero.

Information structure The structure of information can be described with reference to the time line in Figure 1. At the start-up stage nobody knows the project's potential profitability $\theta$. We assume that $\theta$ is a random variable with bounded support $\Theta \subset \Re_{++}$and cumulative distribution function $F(\theta)$. At the expansion stage $\theta$ becomes observable. The idea is that between the start-up stage and the expansion stage some crucial information about the project gets revealed. This information may be verifiable and thus incorporated as a contingency in the initial financial contract, or not verifiable and thus noncontractible. We will consider and compare both scenarios. 


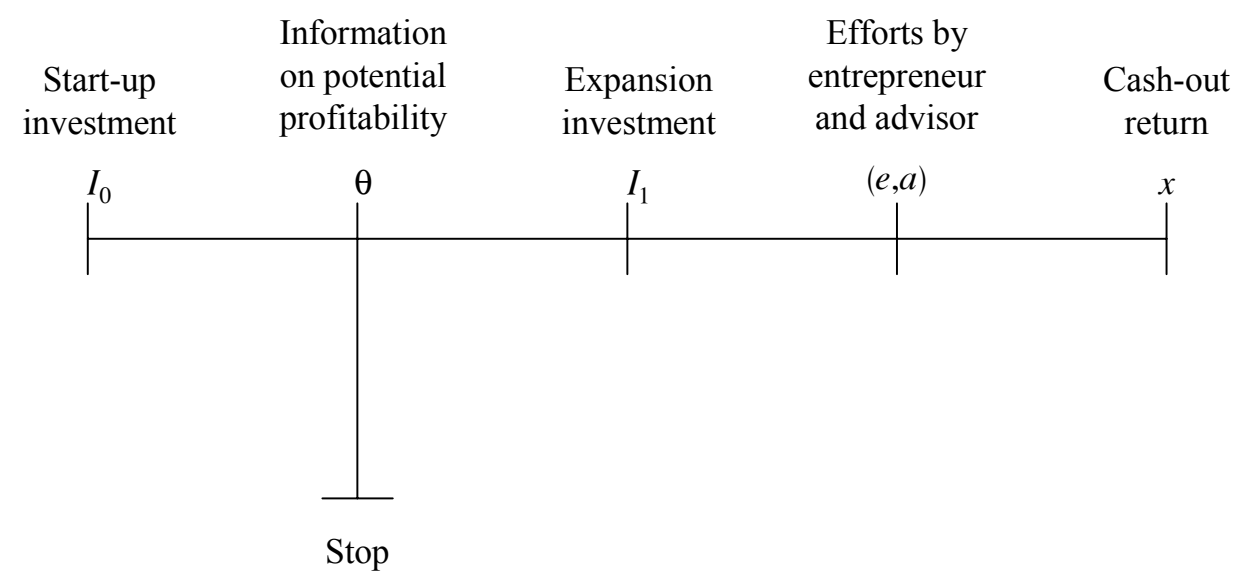

Figure 1: The sequence of events

The efforts $e$ and $a$ are contributed during the expansion stage provided that the investment $I_{1}$ has been made. As in standard moral hazard models, $e$ and $a$ are unobservable except to the agent who exerts them. Finally, the realization of $x$ at the cash-out stage (whenever reached) is assumed to be observable and verifiable.

With this timing of events we intend to focus on the incentive problems that arise in the expansion stage. Informal descriptions of venture capitalists' advising role suggest that their input is indeed more important for the development and marketing of well-defined products (expansion stage) than for the construction of first prototypes (start-up stage). Entrepreneurs' activities seem, in principle, crucial in both stages. Yet, one can argue that focusing on the expansion stage makes sense since, during the start-up stage, the incentive to guarantee the continuation of the project on to the next stage (say, by exerting effort in order to shift the distribution of $\theta$ towards the right) is, in most cases, sufficient to keep the entrepreneurs' moral hazard problem under control.

Contracting possibilities The relevant contingencies in the life of the project after the initial investment $I_{0}$ are, by chronological order, the realization of the project's potential profitability $\theta$, the investment of $I_{1}$, the effort contributions $e$ and $a$, and the realization of $x$. Clearly, the unobservability of $e$ and $a$ impedes writing contracts contingent on these variables. 
When $\theta$ is verifiable, some initial long-term contract between the entrepreneur and one or several venture capitalists (of which one acts as an advisor) can be signed so as to establish the terms for the provision of $I_{0}$ and, contingent on $\theta$, for the provision of $I_{1}$. We consider the optimal design of this contingent financing contract in Section 4 .

When $\theta$ is not verifiable, the initial contract cannot make the provision of the funds $I_{1}$ directly contingent on $\theta$. Our analysis focuses on the empirically relevant case of stage financing whereby funds are raised as they are needed. In particular, in Section 5 we consider the case in which a contract signed at the start-up stage promises, in exchange for $I_{0}$, a share of $x$ if the project reaches the cash-out stage. After the realization of $\theta$, the entrepreneur tries to arrange the financing of $I_{1}$ (and the provision of $a$ ), renegotiating, if convenient, the contract signed for the financing of $I_{0}$. The alternative polar arrangement would be upfront financing whereby the entrepreneur raises $I_{0}+I_{1}$ at the start-up stage, invests $I_{0}$, and keeps $I_{1}$ so as to decide on the expansion investment after observing $\theta$. At that point she can invest $I_{1}$ in the project, or share it with her financiers according to some pre-specified liquidation rule. $^{7}$ Additionally, there might be intermediate arrangements in which only a part of $I_{1}$ is raised upfront. ${ }^{8}$ In Section 6.1 we discuss the conditions under which stage financing dominates the arrangements in which $I_{1}$ is partly or fully raised upfront.

In all the cases we assume limited liability, so that all terminal payments must be nonnegative. Hence when the project is abandoned and also when it continues and fails, the entrepreneur and her financiers do not receive or pay anything, since the final return is zero. In contrast, when the project is continued and succeeds, $\theta$ is divided up among the parties. ${ }^{9}$

\footnotetext{
${ }^{7}$ Stage financing is commonly justified by invoking the existence of sizeable private benefits of control for the entrepreneur. As Sahlman (1990, pp. 506-507) puts it: "By staging capital the venture capitalists preserve the right to abandon a project whose prospects look dim. The right to abandon is essential because an entrepreneur will almost never stop investing in a failing project as long as others are providing capital." Clearly, with excessive continuation, if low realizations of $\theta$ are sufficiently likely and/or the expansion investment $I_{1}$ is sufficiently large, upfront financing may not be feasible.

${ }^{8}$ Neher (1999) endogenizes the staging of finance when a hold-up problem limits the repayment capacity of the entrepreneur, which depends on the liquidation value of the venture.

${ }^{9}$ We rule out budget-breaking schemes of the sort analyzed by Holmström (1982). In these schemes a third party (say, another venture capitalist) pays a bonus to the entrepreneur and her advisor when the project succeeds, and is compensated by an initial transfer from the venture capitalist who acts as an advisor. A well-known problem with these schemes is that they are not in
} 
Strategy for the analysis Our model has two main building blocks: the doublesided moral hazard problem with wealth constraints and the staging of investment and finance. In order to highlight the trade-offs introduced by the first building block, Section 3 focuses on a simplified version of the model in which the financing problem only arises once the project's potential profitability $\theta$ is publicly known. The impact of the financing needs of the start-up stage is then analyzed in Sections 4 and 5, along the lines mentioned above.

\section{Financing the Expansion Stage}

We start considering a model in which the entrepreneur must raise $I_{1}$ and recruit a venture capitalist with whom to pursue the successful development of a project whose potential profitability $\theta$ is publicly known. We assume that no outstanding claim on the success return exists at this point, so $\theta$ can be used in full as compensation for the required funds and efforts. The entrepreneur tries to obtain the funds $I_{1}$ and the required advice by offering a contract to one or several venture capitalists. ${ }^{10}$

In Appendix A we show that the optimal contract involves a single venture capitalist as the sole financier of the project. Intuitively, because of the double-sided moral hazard problem, the whole success return $\theta$ is useful for providing incentives to the entrepreneur and her advisor. Given that venture capitalists are assumed to be wealthy, the one chosen to act as an advisor can optimally afford to finance the entire investment $I_{1} \cdot{ }^{11}$ For this reason, we can hereafter restrict attention to contracts between the entrepreneur and a venture capitalist.

A contract between an entrepreneur (E) and a venture capitalist (V) is a pair $(s, T)$ that specifies:

(i) the share $s \in[0,1]$ of the success return $\theta$ that is given to $\mathrm{V}$, and

(ii) an initial transfer $T \geq 0$ that $\mathrm{E}$ receives from $\mathrm{V}$.

general robust to collusion (see, for example, Eswaran and Kotwal, 1984).

${ }^{10}$ This situation corresponds to the particular case of the general model in which $I_{0}=0$ and the entrepreneur starts up the project without a precommitment of funding or provision of advice by any other agent.

${ }^{11}$ The formal proof of the result is based on showing that had another venture capitalist been given a share of $\theta$, this should be repurchased by the one acting as an advisor, generating (through its positive effect on efforts) a higher payoff for both him and the entrepreneur. 
The transfer $T$ ensures that all the surplus of the project is appropriated by the entrepreneur - a result that follows from ex ante competition among venture capitalists. Accordingly, if incentive reasons make it desirable to give $\mathrm{V}$ a share $s$ of $\theta$ which leads him to obtain a payoff (net of the disutility of his effort) that exceeds $I_{1}$, then E can be compensated for the difference by means of a positive transfer $T$. In contrast, if allocating effort more efficiently required a large entrepreneurial share, $1-s$, there may be a conflict with V's individual rationality constraint, since the wealth-constrained entrepreneur can only compensate him through $s$ (that is, $T<0$ is not feasible). As a result, finance-related distortions in the provision of effort may occur.

For a given success return $\theta$, a contract $(s, T)$ defines a simultaneous move game between $\mathrm{E}$ and $\mathrm{V}$, whose payoff functions are, respectively,

$$
\begin{aligned}
& p(e, a)(1-s) \theta-U(e)+T \\
& p(e, a) s \theta-V(a)-I_{1}-T
\end{aligned}
$$

Clearly $p(e, 0)=p(0, a)=0$ implies that $(e, a)=(0,0)$ is always a Nash equilibrium of this game. But under this equilibrium the project fails with probability one and, hence, is not viable. However there exists a second Nash equilibrium which is characterized in the following result.

Lemma 1 For any $\theta \in \Theta$ and any contract $(s, T)$, the game between $E$ and $V$ always has a unique equilibrium with positive levels of effort. Moreover if the upper bound of $\Theta$ is not too large this equilibrium is always interior. The corresponding probability of success is

$$
p(s, \theta)=\rho(s) \theta
$$

and the equilibrium payoffs of $E$ and $V$ are

$$
\begin{aligned}
& \Pi_{E}(s, \theta)+T=\frac{1}{2}(1+\alpha)(1-s) \rho(s) \theta^{2}+T, \\
& \Pi_{V}(s, \theta)-T=\frac{1}{2}(2-\alpha) s \rho(s) \theta^{2}-I_{1}-T,
\end{aligned}
$$

where

$$
\rho(s)=[u(1-\alpha)(1-s)]^{1-\alpha}(v \alpha s)^{\alpha} .
$$


It should be noted that while $\Pi_{E}(s, \theta)$ is always nonnegative (since by choosing $e=0$ the entrepreneur can get a zero payoff), $\Pi_{V}(s, \theta)$ may be negative, but (by the same argument) it is never smaller than $-I_{1}$. Adding up the payoffs of $\mathrm{E}$ and $\mathrm{V}$ gives the net continuation return of the project:

$$
\Pi(s, \theta)=\Pi_{E}(s, \theta)+\Pi_{V}(s, \theta)=\frac{1}{2}(1+\alpha+s-2 \alpha s) \rho(s) \theta^{2}-I_{1} .
$$

Clearly, $\Pi_{E}(s, \theta) \geq 0$ implies that $\Pi(s, \theta) \geq \Pi_{V}(s, \theta)$.

The optimal contract for a given $\theta$, denoted $(s(\theta), T(\theta))$, maximizes the equilibrium payoff of $\mathrm{E}$

$$
\Pi_{E}(s, \theta)+T
$$

subject to V's individual rationality constraint

$$
\Pi_{V}(s, \theta)-T \geq 0
$$

and E's wealth constraint

$$
T \geq 0
$$

Clearly (7) will be satisfied with equality. Hence substituting $T=\Pi_{V}(s, \theta)$ into (6) and (8) gives the following compact definition of the optimal contract:

$$
s(\theta)=\arg \max _{s}\left\{\Pi(s, \theta) \mid \Pi_{V}(s, \theta) \geq 0\right\}
$$

and $T(\theta)=\Pi_{V}(s(\theta), \theta)$.

In order to characterize this contract we first state a result that summarizes the properties of $\Pi(s, \theta)$ and $\Pi_{V}(s, \theta)$.

Lemma 2 The functions $\Pi(s, \theta)$ and $\Pi_{V}(s, \theta)$ are quasiconcave in $s$, and satisfy

$$
\Pi(0, \theta)=\Pi(1, \theta)=\Pi_{V}(0, \theta)=\Pi_{V}(1, \theta)=-I_{1} .
$$

Moreover, $\Pi(s, \theta)$ reaches a maximum for $s^{*} \in\left(\alpha, \frac{1}{2}\right)$ if $\alpha<\frac{1}{2}, s^{*} \in\left(\frac{1}{2}, \alpha\right)$ if $\alpha>\frac{1}{2}$, and $s^{*}=\frac{1}{2}$ if $\alpha=\frac{1}{2} ; \Pi_{V}(s, \theta)$ reaches a maximum for $\widehat{s}=\frac{1}{2}(1+\alpha)>s^{*}$.

The functions $\Pi(s, \theta)$ and $\Pi_{V}(s, \theta)$ are depicted in Figure 2 (for fixed $\left.\theta\right)$. The net continuation return $\Pi(s, \theta)$ reaches a maximum for a share $s^{*}$ lower than the share $\widehat{s}$ that maximizes V's payoff $\Pi_{V}(s, \theta)$. In the optimal contract problem, $\Pi(s, \theta)$ is the 


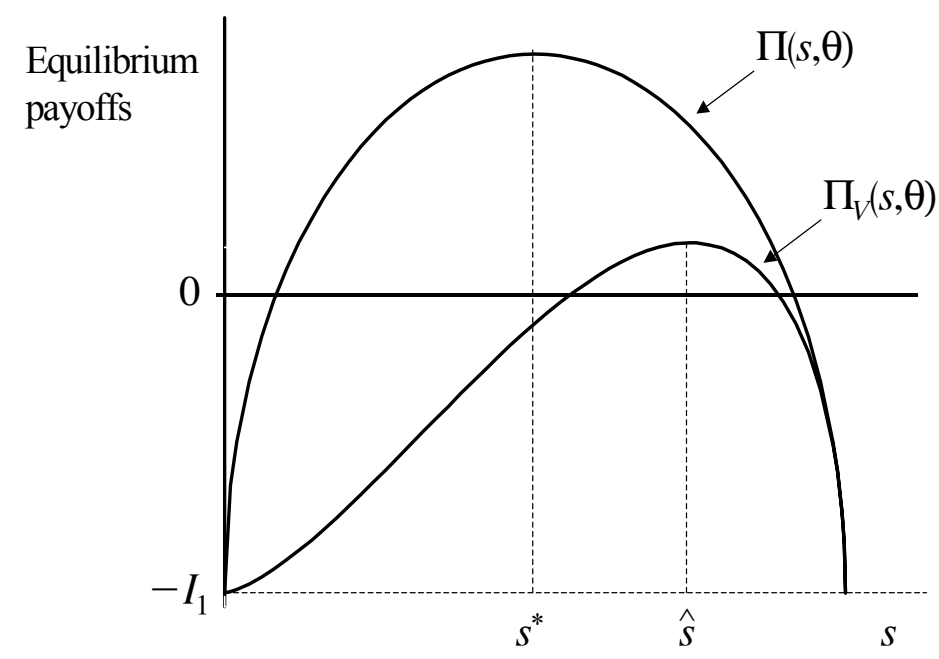

Figure 2: Equilibrium payoffs of the entrepreneur and the venture capitalist

function to be maximized, while $\Pi_{V}(s, \theta) \geq 0$ represents the financing constraint. Hence, if $\Pi_{V}\left(s^{*}, \theta\right) \geq 0$ the financing constraint is not binding, and the optimal share is simply $s^{*}$. If $\Pi_{V}\left(s^{*}, \theta\right)<0 \leq \Pi_{V}(\widehat{s}, \theta)$ (the case shown in Figure 2) there is an interval of sharing rules around $\widehat{s}$ that satisfy the constraint. Since this interval is to the right of $s^{*}$, where $\partial \Pi(s, \theta) / \partial s<0$, it follows that $\Pi(s, \theta)$ is maximized at the smallest feasible $s$. Finally, the project is not feasible if $\Pi_{V}(\widehat{s}, \theta)<0$. Only in the first of these cases the transfer from $\mathrm{V}$ to $\mathrm{E}$ is positive, with $T(\theta)=\Pi_{V}\left(s^{*}, \theta\right)$.

To analyze how these possibilities relate to the project's potential profitability $\theta$, notice from (3) that the functions $\Pi_{V}(\widehat{s}, \theta)$ and $\Pi_{V}\left(s^{*}, \theta\right)$ are increasing in $\theta$ and satisfy $\Pi_{V}(\widehat{s}, 0)=\Pi_{V}\left(s^{*}, 0\right)=-I_{1}$. Moreover, by the definition of $\widehat{s}$ we have $\Pi_{V}(\widehat{s}, \theta)>$ $\Pi_{V}\left(s^{*}, \theta\right)$ for all $\theta>0$. Hence we can state the following result.

Proposition 1 There are two critical values $\hat{\theta}$ and $\theta^{*}$, with $\hat{\theta}<\theta^{*}$, such that

(i) if $\theta<\widehat{\theta}$ the project is not feasible,

(ii) if $\widehat{\theta} \leq \theta<\theta^{*}$ the optimal contract is $s(\theta)=\min \left\{s \mid \Pi_{V}(s, \theta)=0\right\}$ and $T(\theta)=0$,

(iii) if $\theta \geq \theta^{*}$ the optimal contract is $s(\theta)=s^{*}$ and $T(\theta)=\Pi_{V}\left(s^{*}, \theta\right)$. 
Differentiating the equation $\Pi_{V}(s, \theta)=0$ that implicitly defines $s(\theta)$ for $\widehat{\theta} \leq \theta<$ $\theta^{*}$, one can immediately obtain that $s^{\prime}(\theta)<0 .{ }^{12}$ Thus, as the potential profitability of the project $\theta$ increases, the distortions required for compensating the venture capitalist decline: in particular, V's share $s(\theta)$ becomes closer (and eventually equal) to the share $s^{*}$ that maximizes the net continuation return of the project. ${ }^{13}$

The double-sided moral hazard problem analyzed in this section provides an important building block for our general model. We have shown how incentive concerns justify the exclusive provision of finance by the venture capitalist who contributes his advice to the firm. This allows the project's success return to be entirely used for incentive purposes. When profitability prospects are sufficiently good $\left(\theta \geq \theta^{*}\right)$, the financing constraint is not binding and the optimal share of the venture capitalist solely depends on the technology and cost of effort parameters. In contrast, for poorer prospects $\left(\widehat{\theta} \leq \theta<\theta^{*}\right)$, the financing constraint is binding and the optimal share of the venture capitalist is distorted up and away from its unconstrained value, becoming sensitive to the size of the required investment (that pushes it further up). The distortions caused by financing burdens are a crucial consideration in the analysis that follows.

\section{Contingent Financing}

Suppose now that the project requires some start-up investment $I_{0}$ before the information on its potential profitability $\theta$ is revealed. In this section we assume that $\theta$ is verifiable, so that the entrepreneur and her venture capitalist can sign a long-term contract that establishes the conditions for the funding of $I_{1}$ at the same time as the funding of $I_{0}$ is arranged. This case covers situations in which the information on a project's potential profitability $\theta$ can be expressed in the form of some objective performance indicators or milestones. Additionally, this case provides us with a

\footnotetext{
${ }^{12}$ Since $\partial \Pi_{V}(s, \theta) / \partial \theta>0$ and, for $s=s(\theta)<\widehat{s}$, we have $\partial \Pi_{V}(s, \theta) / \partial s>0$.

${ }^{13}$ These results can be put into perspective by looking at two limit cases that have been frequently studied in the corporate finance literature. The typical entrepreneurial moral hazard model with financing constraints emerges when $\mathrm{V}$ has no role as a provider of effort $(\alpha=0)$. In this case, we have $s^{*}=0$ so $\mathrm{V}$ is given the minimum share $s(\theta) \in(0, \widehat{s}]$ that compensates him for $I_{1}$. In contrast, if only $\mathrm{V}$ has a role as a provider of effort $(\alpha=1)$, we have $s^{*}=\widehat{s}=1$ so the project must become $100 \%$ owned (and managed) by V. In such a case, the optimal contract sets $s(\theta)=1$ and E profits from the project by selling it to $\mathrm{V}$ in exchange for the transfer $T(\theta)=\Pi_{V}(1, \theta)$.
} 
benchmark against which to evaluate the effects and the costs of the (arguably more realistic) non-verifiability of $\theta$, that we study in the next section.

In the verifiable case, a venture capitalist provides the start-up investment $I_{0}$ and commits to provide the expansion investment $I_{1}$ for the contractually specified continuation values of $\theta$. A contingent financing contract between $\mathrm{E}$ and $\mathrm{V}$ specifies:

(i) a cutoff point $\bar{\theta}$ below which the project is abandoned,

(ii) the share $z(\theta)$ of the success return $\theta$ (for $\theta \geq \bar{\theta}$ ) that is given to $\mathrm{V}$, and

(iii) an initial transfer $T \geq 0$ that $\mathrm{E}$ receives from $\mathrm{V}$.

Using our previous definitions of the equilibrium payoffs of $\mathrm{E}$ and $\mathrm{V}$ in the efforts game (see Lemma 1), the optimal contingent contract maximizes the expected payoff of $\mathrm{E}$

$$
\int_{\bar{\theta}}^{\infty} \Pi_{E}(z(\theta), \theta) d F(\theta)+T
$$

subject to V's individual rationality constraint

$$
\int_{\bar{\theta}}^{\infty} \Pi_{V}(z(\theta), \theta) d F(\theta) \geq I_{0}+T
$$

and E's wealth constraint

$$
T \geq 0
$$

Clearly (12) will be satisfied with equality. Hence solving for $T$ in this expression, substituting it into (11) and (13), and using the definition (5) of the net continuation return of the project gives the following equivalent problem:

$$
\max _{(\bar{\theta}, z(\theta))} \int_{\bar{\theta}}^{\infty} \Pi(z(\theta), \theta) d F(\theta)-I_{0},
$$

subject to

$$
\int_{\bar{\theta}}^{\infty} \Pi_{V}(z(\theta), \theta) d F(\theta) \geq I_{0}
$$

In words, the optimal contingent contract maximizes the project's net present value subject to the constraint that V's expected payoff suffices to compensate him for the funding of the start-up investment $I_{0} \cdot{ }^{14}$

The optimal contract when $\theta$ is verifiable is characterized in the following result.

\footnotetext{
${ }^{14}$ Although in the previous expressions we integrate between $\bar{\theta}$ and $\infty$, it must be noted that the support $\Theta$ of $\theta$ is assumed to be bounded.
} 
Proposition 2 When the information on the project's potential profitability $\theta$ is verifiable, there are two critical values $L$ and $H$, with $L<H$, such that

(i) if $I_{0}>H$ the project is not feasible,

(ii) if $L<I_{0} \leq H$ the optimal contract is characterized by a constant share $z(\theta)=$ $\bar{s} \in\left(s^{*}, \widehat{s}\right]$ for all $\theta \geq \bar{\theta}$, where the cutoff point $\bar{\theta}$ is such that $\bar{\theta} \leq \widehat{\theta}$, and $T=0$,

(iii) if $I_{0} \leq L$ the optimal contract is characterized by a constant share $z(\theta)=s^{*}$ for all $\theta \geq \bar{\theta}$, where the cutoff point $\bar{\theta}$ is such that $\bar{\theta}<\widehat{\theta}$ and satisfies $\Pi\left(s^{*}, \bar{\theta}\right)=0$, and $T=L-I_{0}$.

This result reflects the tension between maximizing the net present value of the project and ensuring that its financing is feasible. When the start-up investment is too large $\left(I_{0}>H\right)$, financing the project is not feasible, even if the share $z(\theta)$ given to $\mathrm{V}$ is set equal to the value $\widehat{s}$ that maximizes his net continuation payoff, and the cutoff point $\bar{\theta}$ is set equal to the value for which $\Pi_{V}(\widehat{s}, \theta)$ is zero. Conversely, when the start-up investment is sufficiently small $\left(I_{0} \leq L\right)$, the project can be financed under the share $s^{*}$ that maximizes the project's net continuation return, and with the cutoff point $\bar{\theta}$ equal to the value for which $\Pi\left(s^{*}, \theta\right)$ is zero. For intermediate values of $I_{0}$, financing the project is feasible, but requires a (constant) share $\bar{s}$ which is distorted away from $s^{*}$ and approaches $\widehat{s}$ as the start-up investment increases.

Two interesting features distinguish this optimal contingent contract from the profile of expansion stage contracts characterized in Proposition 1. First, the share $z(\theta)$ is constant while the share $s(\theta)$ is (weakly) decreasing in $\theta$. Second, if $I_{0}<H$ there is a range of states, $[\bar{\theta}, \widehat{\theta})$, in which the optimal contingent contract allows the project to be continued despite the fact that V's maximum continuation payoff is negative - that is, despite the fact that funding the investment $I_{1}$ with an expansion stage contract would be impossible. These two features, which are depicted in Figure 3 , reflect how V's precommitment to the state-contingent funding of the expansion investment $I_{1}$ allows the high-profitability states to "subsidize" some of the lowprofitability states. Such subsidization contributes to minimize the distortions to the incentive problem of the expansion stage (reflected in the need for $\bar{s}>s^{*}$ ) and prevents the discontinuation of some projects that are overall valuable but would not be financially viable otherwise (reflected in the fact that $\Pi_{V}(\bar{s}, \bar{\theta})<0<\Pi(\bar{s}, \bar{\theta})$ ). 


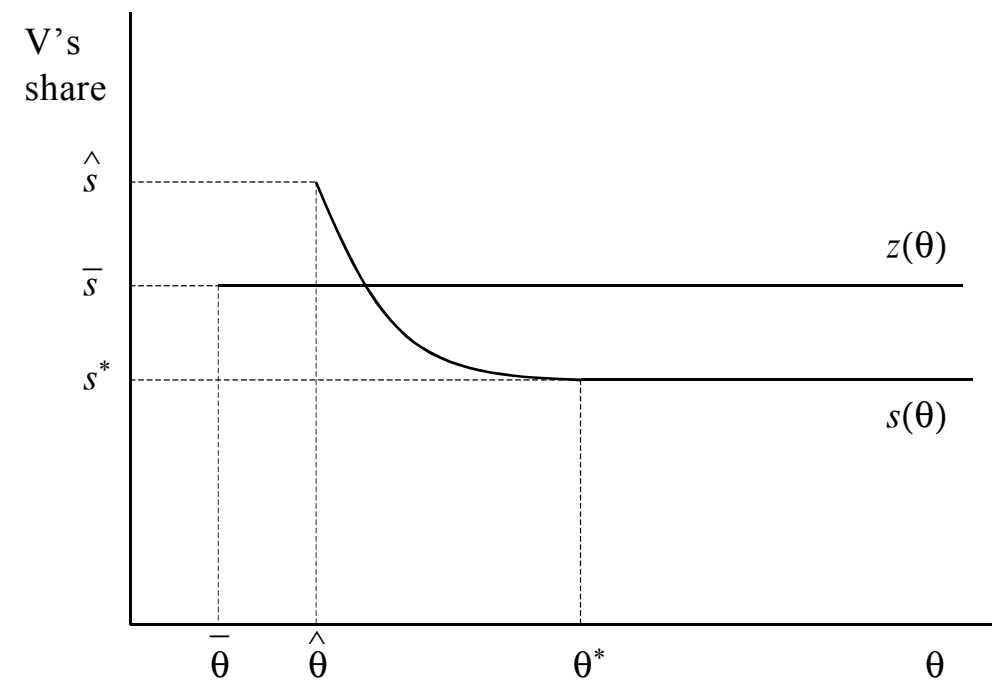

Figure 3: The venture capitalist's share under contingent financing

In the next section we show that when $\theta$ is not verifiable, implementing this subsidization is more complicated, since the terms for the continuation of the project cannot be directly written into the initial contract between the entrepreneur and her venture capitalist. Nevertheless this contract will still be structured so as to minimize the distortions imposed by the financing of the start-up investment.

\section{$5 \quad$ Stage Financing}

The project's potential profitability $\theta$ is now assumed to be observable but, in contrast to the previous section, not verifiable. This prevents any state-contingent precommitment to fund the expansion stage. Hence we consider a situation in which the financing of the start-up investment $I_{0}$ and the financing of the expansion investment $I_{1}$ are arranged sequentially.

Funding $I_{0}$ requires pledging a share $r(\theta) \in[0,1]$ of the final success return at the beginning of the start-up stage, while funding $I_{1}$ requires pledging some additional share of such return at the beginning of the expansion stage. Strictly speaking we should write the share $r$ as a function of the final verifiable return $x$. But since the 
project can either succeed or fail, and the return in case of failure is zero, we will simply write $r(\theta)$ to describe, with a slight abuse of notation, V's initial claim.

In modelling the stage financing process, we assume that funding the start-up investment gives no exclusivity rights for the funding of the expansion investment, ${ }^{15}$ and we allow for the renegotiation of the start-up contract at the beginning of the expansion stage. In other words, the position of the initial venture capitalist $\mathrm{V}$ is "contestable" in that he faces the potential competition from other venture capitalists at all stages of the life of the project. ${ }^{16}$ For the start-up stage, this competition reduces V's net expected payoff to zero. For the expansion stage, we assume that V has all the bargaining power in the renegotiation with $\mathrm{E}$, so the latter would be indifferent between continuing with the former or finding a different venture capitalist for the expansion stage.

Assuming that the venture capitalist has all the bargaining power is consistent with the common wisdom that professional financiers are "strong" relative to the entrepreneurs. One reason for this strength may be that financiers are repeatedly involved in debt or more generally contract renegotiations, which gives them an incentive to develop a reputation as tough bargainers. In particular, dynamic reputational concerns may allow them to sustain bargaining strategies involving "threats" that would not be credible in a one-shot bargaining game. ${ }^{17}$

In order to characterize the optimal structure of the initial claim $r(\theta)$ we proceed in an indirect manner. The renegotiation of any initial claim at the beginning of the expansion stage leads $\mathrm{V}$ to obtain some net continuation payoff $C(\theta)$. The design of $r(\theta)$ must take into account the distortions that the implied $C(\theta)$ causes during the expansion stage. It turns out that any relevant net continuation payoff can be implemented by some properly chosen initial claim. This allows us to solve the security design problem in two stages. First we derive the profile $C(\theta)$ that maximizes

\footnotetext{
${ }^{15}$ Such feature would imply that in all continuation states the venture capitalist's share would be the value $\widehat{s}$ that maximizes $\Pi_{V}(s, \theta)$. The associated distortion in the allocation of effort would in general reduce the entrepreneur's expected payoff in such a way that another venture capitalist could undercut the initial exclusive contract.

${ }^{16}$ This assumption is reasonable if the information on the project's potential profitability $\theta$ can be costlessly observed by outside venture capitalists at the beginning of the expansion stage. We consider as an extension in Section 6.2 the case in which these venture capitalists have to incur a cost to observe $\theta$.

${ }^{17}$ We consider as an extension in Section 6.3 the case in which E has some bargaining power.
} 
E's expected payoff subject to the constraint that $\mathrm{V}$ is compensated for his funding of the start-up investment $I_{0}$. Then we analyze the renegotiation between $\mathrm{E}$ and $\mathrm{V}$ after $\theta$ is observed, and derive the initial claim $r(\theta)$ that implements the optimal $C(\theta)$.

\subsection{Distributing the burden of the start-up investment}

Confirming the intuitions developed in previous sections, the incentive costs of compensating $\mathrm{V}$ for funding the start-up investment $I_{0}$ are larger when the project's potential profitability $\theta$ is low. For this reason, as we show below, it is optimal to set $C(\theta)$ equal to zero in the lowest profitability states and thereafter, by the same logic as in the case of contingent financing, to structure $C(\theta)$ so as to induce a constant sharing of the success return.

Formally, the optimal expansion stage contract that gives the venture capitalist a net continuation payoff $C \geq 0$ is

$$
s(\theta, C)=\arg \max _{s}\left\{\Pi(s, \theta)-C \mid \Pi_{V}(s, \theta) \geq C\right\} .
$$

The only difference with respect to the expansion stage contract (9) is that now $\mathrm{V}$ must be compensated for $C$ in addition to $I_{1}$ and the cost of his advising effort. Hence the solution satisfies $s(\theta, 0)=s(\theta)$ and $s(\theta, C) \geq s(\theta)$. And clearly, except when $\theta>\theta^{*}$ and $C$ is sufficiently small, we have $s(\theta, C)>s(\theta)$, so the presence of $C$ distorts V's share strictly above $s(\theta)$.

The optimal compensation of $\mathrm{V}$ is then obtained by solving the following problem:

$$
\max _{C(\theta)} \int_{\widehat{\theta}}^{\infty}[\Pi(s(\theta, C(\theta)), \theta)-C(\theta)] d F(\theta)
$$

subject to V's individual rationality constraint

$$
\int_{\widehat{\theta}}^{\infty} C(\theta) d F(\theta)=I_{0}
$$

and the nonnegativity constraint

$$
C(\theta) \geq 0
$$

The objective function of this problem reflects that in each continuation state, $\theta \geq \widehat{\theta}$, $\mathrm{E}$ will appropriate the difference between the net continuation return of the project, 
$\Pi(s(\theta, C(\theta)), \theta)$, and V's compensation, $C(\theta)$. Clearly, the lower bound of the continuation region is $\widehat{\theta}$ (i.e. the value for which $\max _{s} \Pi_{V}(s, \widehat{\theta})=\Pi_{V}(\widehat{s}, \widehat{\theta})=0$ ), since for $\theta<\widehat{\theta}$ financing just the expansion investment is already not feasible. ${ }^{18}$

Substituting (18) into (17), the optimal compensation problem can be restated as maximizing the net present value of the project

$$
\max _{C(\theta)} \int_{\widehat{\theta}}^{\infty} \Pi(s(\theta, C(\theta)), \theta) d F(\theta)-I_{0},
$$

subject to the constraints (18) and (19). The following result characterizes the solution to this problem.

Proposition 3 When the information on the project's potential profitability $\theta$ is not verifiable, there are two critical values $L^{\prime}$ and $H$, with $L^{\prime}<H$, such that

(i) if $I_{0}>H$ the project is not feasible,

(ii) if $L^{\prime}<I_{0} \leq H$ the optimal compensation is

$$
C(\theta)= \begin{cases}0, & \text { if } \theta \leq \widetilde{\theta}, \\ \Pi_{V}(\widetilde{s}, \theta), & \text { otherwise }\end{cases}
$$

where $\widehat{\theta} \leq \widetilde{\theta}<\theta^{*}$ and $\widetilde{s}=s(\widetilde{\theta})$,

(iii) if $I_{0} \leq L^{\prime}$ any compensation $C(\theta)$ that satisfies (18) with $C(\theta)=0$ for $\theta \leq \theta^{*}$ and $0 \leq C(\theta) \leq \Pi_{V}\left(s^{*}, \theta\right)$ for $\theta>\theta^{*}$ is optimal.

Intuitively, the size of the start-up investment $I_{0}$ determines the compensation $C(\theta)$ (which turns out to the infeasible if $I_{0}$ is too large) and the severity of its associated distortions (which turn out to be nil if $I_{0}$ is sufficiently small). For intermediate values of $I_{0}$, the optimal compensation $C(\theta)$ is structured so as to make V's final share, $s(\theta, C(\theta))=\max [s(\theta), \widetilde{s}]$, as close as possible to a constant - the type of final share that prevails under contingent financing.

The share $s(\theta, C(\theta))$ is depicted in Figure 4: the decreasing section $s(\theta)$ (for $\widehat{\theta} \leq \theta \leq \widetilde{\theta})$ coincides with the expansion stage share defined by (9), while the flat

\footnotetext{
${ }^{18}$ The optimal compensation problem should also include constraints on the values of $C(\theta)$ that can be implemented by renegotiating an initial claim $r(\theta)$. However, we show in the next subsection that these constraints are in general never binding, so we can ignore them.
} 


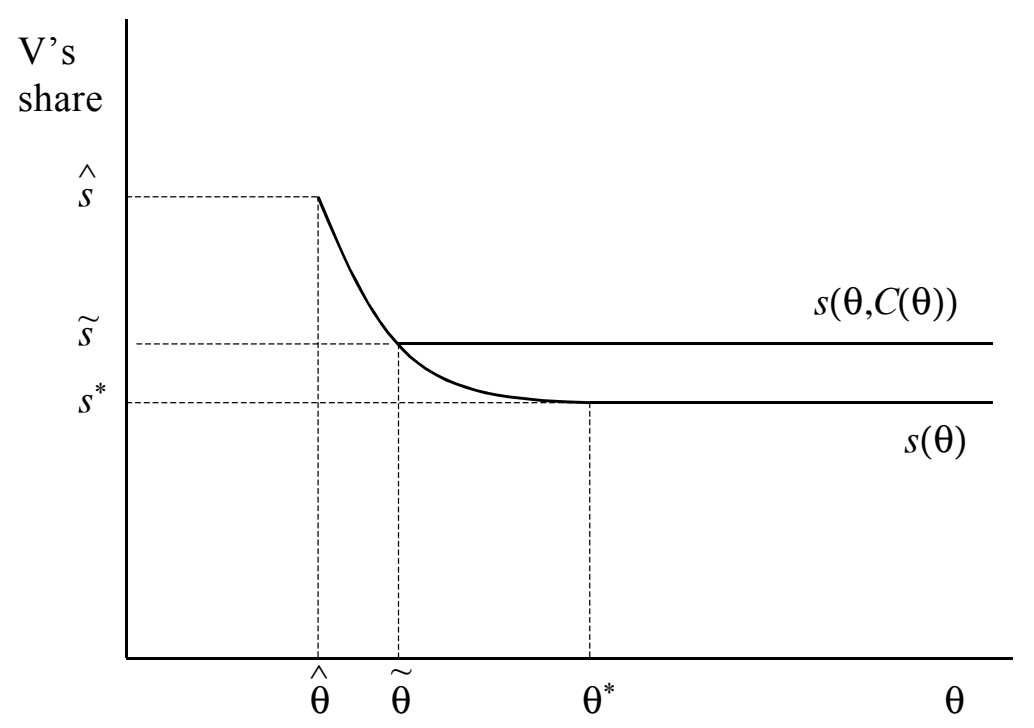

Figure 4: The venture capitalist's final share under stage financing

section $\widetilde{s}$ shifts down (up) towards $s^{*}(\widehat{s})$ as $I_{0}$ decreases (increases) towards $L^{\prime}(H)$. In contrast with the case of contingent financing (see Figure 3), with stage financing it is not possible to "subsidize" the low-profitability states with the returns of the high-profitability states and, hence, it is not possible to implement a constant share over all continuation states.

From here it follows that the constant share $\bar{s}$ associated with the optimal contingent contract is larger than the share $\widetilde{s} .{ }^{19}$ For the same reason, a somewhat paradoxical implication follows: the critical value $L^{\prime}$ is larger than $L$ and, therefore, the share $s^{*}$ that maximizes the net continuation return of the project will tend to be more prevalent under stage financing than under contingent financing. ${ }^{20}$

\footnotetext{
${ }^{19}$ To see this, notice that $\bar{s}$ and $\widetilde{s}$ satisfy $\int_{\bar{\theta}}^{\infty} \Pi_{V}(\bar{s}, \theta) d F(\theta)=\int_{\tilde{\theta}}^{\infty} \Pi_{V}(\widetilde{s}, \theta) d F(\theta)=I_{0}$. But then $\bar{\theta}<\widehat{\theta}<\widetilde{\theta}$ and $\Pi_{V}(\widetilde{s}, \theta)<0$ for $\theta<\widetilde{\theta}$ imply $\bar{s}>\widetilde{s}$.

${ }^{20}$ To check that $L^{\prime}>L$, it suffices to compare expressions (38) and (41) in Appendix B, taking into account that $\bar{\theta}<\widehat{\theta}<\theta^{*}$ and $\Pi_{V}\left(s^{*}, \theta\right)<0$ for $\theta<\theta^{*}$.
} 


\subsection{Bargaining and the initial contract}

We must now study the implementation of the optimal compensation $C(\theta)$ using an initial claim $r(\theta)$ on the project's success return. As already argued, the bargaining between $\mathrm{E}$ and $\mathrm{V}$ should produce a continuation payoff $C(\theta)$ for $\mathrm{V}$, who will remain as E's financier and advisor during the expansion stage. In general, the outcome of this bargaining will depend on the outside options and the bargaining power of the two parties.

If $\mathrm{E}$ and $\mathrm{V}$ fail to reach an agreement, E's outside option is to resort to another venture capitalist $\left(\mathrm{V}^{\prime}\right)$ in order to obtain the funding and advice needed for the expansion stage. In such a case, $\mathrm{E}$ and $\mathrm{V}^{\prime}$ would find that the share $r(\theta)$ is already pledged to $\mathrm{V}$, so the success return effectively available for compensating them would be just

$$
\theta^{\prime}=(1-r(\theta)) \theta
$$

In all other respects, the situation would be identical to the financing of the expansion stage described in Section 3. Hence, by Proposition 1, if $\theta^{\prime} \geq \widehat{\theta}$ the expansion stage contract would give a share $s\left(\theta^{\prime}\right)$ to $\mathrm{V}^{\prime}$ and a continuation payoff of $\Pi\left(s\left(\theta^{\prime}\right), \theta^{\prime}\right)$ to $\mathrm{E}$, whereas if $\theta^{\prime}<\widehat{\theta}$ the expansion investment could not funded and, consequently, E's continuation payoff would be zero.

Having assumed that the initial venture capitalist V has all the bargaining power, the whole surplus from the renegotiation will be appropriated by him, leaving $\mathrm{E}$ with a continuation payoff equal to the value of her outside option $\Pi\left(s\left(\theta^{\prime}\right), \theta^{\prime}\right)$. Hence, an initial claim $r(\theta)$ will implement the optimal compensation $C(\theta)$, and thus will be optimal, if and only if

$$
\Pi(s(\theta, C(\theta)), \theta)-C(\theta)=\Pi\left(s\left(\theta^{\prime}\right), \theta^{\prime}\right),
$$

for all $\theta \geq \widehat{\theta}$. That is, if E's continuation payoff under an agreement whereby $\mathrm{V}$ remains as her financier and advisor, and receives the compensation $C(\theta)$, equals the value of E's outside option.

Equation (21) together with our previous findings allows us to prove the following result.

Proposition 4 For $L^{\prime}<I_{0} \leq H$, the optimal initial claim $r(\theta)$ is a continuous function that satisfies $r(\theta)=0$ for $\widehat{\theta} \leq \theta \leq \tilde{\theta}$, and $r^{\prime}(\theta)>0$ for $\theta>\tilde{\theta}$. 
Thus, for intermediate values of the start-up investment, V's optimal initial claim $r(\theta)$ is uniquely defined for all the states in which the project is continued $(\theta \geq \widehat{\theta}) .{ }^{21}$ Specifically, $r(\theta)$ is equal to zero for $\theta \leq \widetilde{\theta}$, so the project proceeds on to the expansion stage without any burden associated with the start-up investment. For $\theta>\widetilde{\theta}, r(\theta)$ is positive and grows continuously with $\theta$, so that higher realizations of $\theta$ bear a greater burden of the compensation for the start-up investment. This is just how $r(\theta)$ needs to be in order to give $\mathrm{V}$ the same final share $\widetilde{s}$ over the upper part of the range of continuation states (recall Figure 4).

Summing up, our analysis of stage financing shows the strong influence of the double-sided moral hazard problem on the design of the optimal securities in venture capital finance. It is optimal to define the venture capitalist's initial claim in such a way that for most continuation states he receives a constant final share in the success return of the project. This implies concentrating the burden of the funding of the start-up investment in high profitability states.

Unlike in the case of verifiable information, where the optimal contingent contract directly establishes a constant share over all continuation states, now in low profitability states continuation and, if applicable, the venture capitalist's final share are driven by the sole funding of the expansion investment — which per se imposes a higher share than the one uniformly observed in higher profitability states. In order to guarantee that the final share has this form, the venture capitalist's initial claim will give him a share of the final return which is zero over some range and thereafter is positive and increasing. Of course, nothing prevents such non-linear claim to be directly described in the VC contract. However, in practice, VC contracting relies on combinations of standard securities such as equity, preferred stock, and convertibles. In this respect, it is always feasible to structure a sequence of call options on the project's final return which, with a proper graduation of strike prices, increases the venture capitalist's share as profitability increases, approximating arbitrarily well the optimal initial claim. ${ }^{22}$

\footnotetext{
${ }^{21}$ Clearly, for $\theta<\widehat{\theta}$ the shape of $r(\theta)$ is irrelevant since funding the expansion stage is not feasible.

${ }^{22}$ To induce the required curvature in V's overall share, it may be needed to allocate some of the options to E.
} 


\section{$6 \quad$ Extensions}

The extensions considered in this section deal with the robustness of our results to the introduction of entrepreneurial wealth, relationship-specific information, and a more general distribution of the bargaining power in the interim renegotiations between the entrepreneur and the venture capitalist.

\subsection{Entrepreneurial wealth}

Up to this point we have assumed that E does not have any wealth, so she has to completely rely on external finance to undertake the project. In what follows we consider the case where E has some (small) initial wealth, and we ask whether it would be optimal to invest it in the project. In the case of stage financing we also ask whether it would be better to invest it at the start-up stage or at the expansion stage (or at both stages). To answer these questions we compute the value to $\mathrm{E}$ of one dollar available at either the start-up or the expansion stage. For brevity, we focus on the cases in which financing the start-up investment is feasible but V's individual rationality constraint is binding. ${ }^{23}$

In the case of contingent financing (verifiable $\theta$ ), contributing one dollar of her own wealth to the project would allow E to relax V's individual rationality constraint (15). From the proof of Proposition 2, it is clear that E's payoff would increase by $1+\gamma$, where $\gamma$ is the Lagrange multiplier associated with this constraint, which is positive when the constraint is binding. We can then conclude that E should invest all her initial wealth in the project.

In the case of stage financing (not verifiable $\theta$ ), the characterization of V's optimal compensation $C(\theta)$ in Proposition 3 implies that one dollar available at the start-up stage is worth $1+\lambda$, where $\lambda$ is the Lagrange multiplier associated with V's individual rationality constraint (18). On the other hand, the value of one dollar available at the expansion stage depends on the realization of $\theta$. If $\theta<\widehat{\theta}$, the project is abandoned and so one dollar available at the expansion stage is worth just one dollar. If $\widehat{\theta} \leq \theta<\tilde{\theta}$, from the proof of Proposition 3 such dollar is worth $1+\lambda+\mu(\theta)$, where $\mu(\theta)$ is the multiplier associated with the binding constraint $C(\theta) \geq 0$. Finally, if $\theta \geq \widetilde{\theta}$ this

\footnotetext{
${ }^{23}$ That is, we restrict attention to $L<I_{0} \leq H$ (verifiable $\theta$ ) and to $L^{\prime}<I_{0} \leq H$ (not verifiable $\theta)$.
} 
constraint is not binding, so the value of the dollar is $1+\lambda$. Hence the expected value of one dollar at the expansion stage is

$$
1+\lambda[1-F(\widehat{\theta})]+\int_{\widehat{\theta}}^{\widetilde{\theta}} \mu(\theta) d F(\theta)+\Pi(\widehat{s}, \widehat{\theta}) F^{\prime}(\widehat{\theta}) \frac{\partial \widehat{\theta}}{\partial I_{1}},
$$

where the last term takes into account the fact that E's contribution shifts the cutoff point $\widehat{\theta}$ to the left. Comparing the previous expression with $1+\lambda$ we conclude that E should invest her dollar at the start-up stage if and only if

$$
\lambda F(\widehat{\theta}) \geq \int_{\widehat{\theta}}^{\widetilde{\theta}} \mu(\theta) d F(\theta)+\Pi(\widehat{s}, \widehat{\theta}) F^{\prime}(\widehat{\theta}) \frac{\partial \widehat{\theta}}{\partial I_{1}}
$$

When the start-up investment $I_{0}$ approaches the feasibility bound $H$, the Lagrange multiplier $\lambda$ will be high and $\widetilde{\theta}$ will be close to $\widehat{\theta}$, so that this condition will typically hold. It will also hold if the probability of stopping the project, $F(\widehat{\theta})$, is high. On the other hand, when $I_{0}$ approaches the bound $L^{\prime}$ below which the individual rationality constraint (18) ceases to be binding, $\lambda$ tends to zero, and E should use all her wealth to relax V's individual rationality constraints at the expansion stage. Summing up, we should expect the entrepreneur to provide all her wealth upfront in projects where the start-up investment and/or the probability of discontinuation of the project at the expansion stage are relatively large.

With stage financing, the general principle for the allocation of entrepreneurial wealth is that $\mathrm{E}$ should try to equate the expected marginal value of her wealth in both stages of the life of the project. Obviously, there may be corner solutions in which either all the wealth is invested at the start-up or at the expansion stage. In this latter case, E might benefit from reducing the external financing required at the expansion stage by raising funds in excess of $I_{0}$ when initiating the project. This arrangement will generalize the stage financing case analyzed so far. ${ }^{24}$

\subsection{Relationship-specific information}

An important maintained assumption in our analysis of stage financing is that, despite its non-verifiability, the potential profitability of the project is observable to the firm's

\footnotetext{
${ }^{24}$ Notice, however, that $(22)$ is a sufficient condition for pure stage financing to remain the optimal arrangement.
} 
outsiders, in particular other venture capitalists. We now consider what happens if we relax this assumption.

Now suppose that $\theta$ is private information of the firm's insiders, $\mathrm{E}$ and $\mathrm{V}$, and that $\mathrm{E}$ can make it observable to other venture capitalists by incurring a cost $z>0$. In this case, E's outside option in her bargaining with $\mathrm{V}$ is reduced by $z$, which implies that the optimal compensation characterized in Proposition 3 is not implementable. To see this, notice that the indifference condition (21) that implicitly defines $r(\theta)$ becomes

$$
\Pi(s(\theta, C(\theta)), \theta)-C(\theta)=\Pi\left(s\left(\theta^{\prime}\right), \theta^{\prime}\right)-z .
$$

But then for values of $\theta$ with $C(\theta)=0$ (that is for $\widehat{\theta} \leq \theta \leq \tilde{\theta}$ ) we would have $\Pi(s(\theta), \theta)=\Pi\left(s\left(\theta^{\prime}\right), \theta^{\prime}\right)-z$, which requires $r(\theta)<0$, violating limited liability.

Hence the derivation of the optimal compensation has to take into account an implementation constraint of the form $C(\theta) \geq \underline{C}(\theta)$, where

$$
\underline{C}(\theta)=\min \{C \geq 0 \mid \Pi(s(\theta, C), \theta)-C \geq \Pi(s(\theta), \theta)-z\} .
$$

In words, V's minimum compensation must be such that E's continuation payoff is greater than or equal to what she would obtain by resorting to another venture capitalist (and consequently incurring the cost $z$ ) when $r(\theta)=0$. Clearly, for values of $\theta$ slightly above $\widehat{\theta}$, E always prefers to sign with $\mathrm{V}$ the contract $\widehat{s}$ that maximizes V's payoff rather than finding an alternative venture capitalist, so we have $\underline{C}(\theta)=$ $\Pi_{V}(\widehat{s}, \theta)$. Also for values of $\theta$ sufficiently greater than $\theta^{*}$ one can show that $\underline{C}(\theta)=z$. Given the shape of $C(\theta)$ in Proposition 3 it follows that the implementation constraint will not be binding for large values of $\theta$. But since $\mathrm{V}$ will be getting $\underline{C}(\theta)>0$ for values of $\theta$ in an interval to the right of $\widehat{\theta}$, his individual rationality constraint (18) will imply a smaller compensation for large values of $\theta$ than in the model with observable $\theta$. Hence the corresponding claim $r(\theta)$ in the initial contract will be below the one characterized in Proposition 4.

Intuitively, the information cost $z$ of replacing $\mathrm{V}$ weakens E's bargaining position at the expansion stage. From the start-up stage perspective, however, ex-ante competition among venture capitalists ensures that $\mathrm{V}$ will not obtain any extra profit. The implementation of the optimal compensation scheme will then be achieved by reducing V's initial claim $r(\theta)$. Yet the fact that $r(\theta)$ cannot be set below zero implies that 
the effect of V's informational advantage cannot be fully eliminated in some states. As a result, the contract is less efficient.

\subsection{Bargaining power}

We now extend our analysis of stage financing to the case where $\mathrm{E}$ has some bargaining power in the renegotiation with $\mathrm{V}$ at the beginning of the expansion stage. In this situation, the status quo corresponds to the consequences of a deadlock in which the parties remain at the negotiation table but never reach an agreement, so the project is not continued. Hence the status quo payoffs of both parties are zero. In addition, $\mathrm{E}$ has the outside option of finding an alternative venture capitalist for the expansion stage which, assuming that $\theta$ is costlessly observable to other venture capitalists, is worth $\Pi\left(s\left(\theta^{\prime}\right), \theta^{\prime}\right)$ to her.

Let $C_{\beta}(\theta)$ denote V's renegotiation payoff in a generalized Nash bargaining solution when his bargaining power is $0<\beta<1$. Clearly, for $\theta>\widehat{\theta}$ we have $C_{0}(\theta)=0$ and $C_{1}(\theta)=\Pi_{V}(\widehat{s}, \theta)$. By our discussion in Section 5.2, E's payoff under this solution would be $\Pi\left(s\left(\theta, C_{\beta}(\theta)\right), \theta\right)-C_{\beta}(\theta)$. But since she has an outside option of value $\Pi\left(s\left(\theta^{\prime}\right), \theta^{\prime}\right)$, by the Outside Option Principle her equilibrium payoff will be $\max \left\{\Pi\left(s\left(\theta, C_{\beta}(\theta)\right), \theta\right)-C_{\beta}(\theta), \Pi\left(s\left(\theta^{\prime}\right), \theta^{\prime}\right)\right\} .^{25}$

Clearly, for $\beta$ close to 1 this payoff will be equal to $\Pi\left(s\left(\theta^{\prime}\right), \theta^{\prime}\right)$, and so all our previous results remain unchanged. On the other hand, when $\beta$ is close to 0 the outside option has no effect on the renegotiation. This implies that the compensation $C(\theta)$ derived in Proposition 3 is no longer implementable. In this case, the derivation of the optimal compensation would have to take into account an implementation constraint of the form $C(\theta) \leq C_{\beta}(\theta)$. This constraint would complicate the solution to the optimal contract problem, but it is unlikely to change the basic insights of our analysis.

\footnotetext{
${ }^{25}$ This result is obtained in the context of a noncooperative bargaining model with alternating offers in which one of the players (say E) can quit the negotiations to take up an outside option see, for example, Sutton (1986) or Osborne and Rubinstein (1990, Chapter 3). The Outside Option Principle states that if the value of this option is smaller than the equilibrium payoff of player $\mathrm{E}$ in the game with no outside option, then the option has no effect on the equilibrium outcome. Otherwise, the equilibrium payoff of player $\mathrm{E}$ is the value of her option.
} 


\section{Conclusions}

Our analysis of venture capital (VC) finance has focused on the incentive problems that affect entrepreneurs and venture capitalists in an environment with multiple investment stages. Specifically, the VC security design problem is set up in a context where information about the venture's potential profitability arrives between an initial start-up stage, where some initial investment is required, and a subsequent expansion stage, where some further investment as well as the managerial contributions of the entrepreneur and the venture capitalist are needed. The optimal contractual arrangement is crucially affected by the nature of the interim information about the venture's profitability.

When this information is verifiable - that is, it can be materialized in objective performance indicators or milestones - it is possible to design a fully contingent financing contract. This contract explicitly determines the conditions for the continuation of the venture on to the expansion stage as well as the final sharing of its success return between the entrepreneur and the venture capitalist. We have found that the optimal contract assigns the venture capitalist a share that is independent from the venture's success return and, hence, can be interpreted as a straight equity claim. The size of such share is increasing in both the relative importance of the managerial contribution of the venture capitalist and the size of the investments for which he has to be compensated. Interestingly, there is a range of profitability states in which the expansion investment occurs despite the fact that the venture capitalist's continuation payoff is negative. In this sense, the contract exhibits some cross-subsidization from high to low profitability states.

When the interim information is not verifiable - that is, no relevant milestones can be found - the previous type of arrangement is not feasible. In particular, under stage financing the funds for each investment round are raised as they are needed. In this case, the cross-subsidization that characterizes contingent financing is no longer possible and the continuation on to the expansion stage is solely determined by the venture capitalist's ex-post participation constraint. Yet the security design problem is not trivial, as the financing of the start-up investment requires pledging a share of the success return which will condition the resolution of the incentive problems present at the expansion stage. Such initial claim will be renegotiated at the expansion stage, 
giving raise to the final sharing of the venture's success return. We have shown that the design of the optimal initial claim is directed to make this sharing as close as possible to a constant. In fact, for low profitability states, the sole funding of the expansion investment will already require too large a share and, hence, the optimal initial claim must avoid further distortions by giving nothing to the venture capitalist. At higher profitability states, the final constant share is implementable through an initial claim that is increasing in the venture's success return. ${ }^{26}$ Thus, the initial contract is associated with a non-linear claim that, as we have explained, resembles the payoff structure of a sequence of warrants with increasing strike prices.

From an empirical perspective, this latter prediction may seem at odds with the prevalence of convertible preferred stock in VC financing. Such conclusion would be, however, too hasty since our model incorporates important simplifying assumptions. Relaxing some of these assumptions would make the analysis more complex but would produce results conforming more closely with the empirically observed contracts. For example, we could introduce some nonzero final returns in the events of early liquidation (before expansion) and failure (after expansion). Clearly, the allocation of early liquidation returns would only affect the expansion stage incentives through its impact on the distribution of the burden of the start-up investment. Hence, in order to minimize incentive distortions, it would be optimal to include these returns in the payoff of the initial claim of the venture capitalist. These returns might empirically correspond to the fix payment component of convertible preferred stock.

When the project yields some nonzero returns in the event of failure, the analysis is slightly more complex. Our claim that the entrepreneur and the venture capitalist should share the whole success returns of the project would still apply, but for incentive reasons failure returns should be allocated to a third party, who in exchange would contribute funds to either the start-up or the expansion investment. The role of this third party would then resemble that of the limited partners of a typical venture capital fund, who only provide funds and hold a less risky stake in the returns of the

\footnotetext{
${ }^{26}$ Under alternative specifications of the probability of success and the cost of effort, it may not be optimal that the venture capitalist receives (over a certain range of states) a constant share of the final return of the project. However, the idea of distributing the burden of the various investments in the way that best deals with the underlying incentive problem would still apply. In particular, it would still be the case that, under stage financing, the expansion investment is already a too heavy burden in low profitability states, so the optimal start-up contract should impose no extra burden on them.
} 
portfolio companies than the general partners (our venture capitalists).

Exploring in full detail the relationship between the general and the limited partners of a typical venture capital fund is an important topic for future research. In such research, it would probably be reasonable to assume that the funds of the general partners have a higher opportunity cost, in which case the optimal financial arrangement (including the size of each party's contribution) would reflect an interesting trade-off between incentives and funding costs.

Finally, from an empirical perspective, testing our theory would require a careful consideration of the dynamics of $\mathrm{VC}$ stakes across financing rounds and its relationship with the venture's final performance. To our knowledge, research in this direction is still pending. 


\section{Appendices}

\section{A Optimal financing of the expansion stage}

This Appendix shows that the expansion investment $I_{1}$ should be solely provided by the venture capitalist who acts as advisor. To do this we consider three-party contracts that allow for funding from two venture capitalists: one who acts as a pure financier and another who also acts as advisor. Both provide their funds in exchange for shares of the project's success return. Importantly, we do not require the functional forms of $p(e, a), U(e)$, and $V(a)$ used in the text. We only need to assume that $p(e, a)$ is increasing and concave with $\partial^{2} p / \partial e \partial a>0$, except for $e=0$ or $a=0$ where $p(0, a)=p(e, 0)=0$, and that $U(e)$ and $V(a)$ are increasing and convex.

Formally, a contract between the entrepreneur (E), a venture capitalist acting as a pure financier $(\mathrm{F})$, and a venture capitalist acting also as an advisor $(\mathrm{V})$ is a vector $\left(t, s, I_{F}, I_{V}, T_{F}, T_{V}\right)$ that specifies:

(i) the share $t \in[0,1]$ of the success return $\theta$ that is given to $\mathrm{F}$, and the share $s \in[0,1]$ of the remaining return $(1-t) \theta$ that is given to $\mathrm{V}$,

(ii) the funds $I_{F}$ and $I_{V}$ contributed by $\mathrm{F}$ and $\mathrm{V}$, respectively, in order to finance $I_{1}$, where $I_{F}+I_{V}=I_{1}$, and

(iii) the initial transfers $T_{F}$ and $T_{V}$ that $\mathrm{E}$ receives from $\mathrm{F}$ and $\mathrm{V}$, respectively, where $T_{F}+T_{V} \geq 0$.

As before, the transfers $T_{F}$ and $T_{V}$, together with ex ante competition among venture capitalists, ensure that all the surplus of the project is appropriated by E. ${ }^{27}$

Once the funds $I_{F}$ and $I_{V}$ are invested, only $\mathrm{E}$ and $\mathrm{V}$ play an active role in the project, by contributing $e$ and $a$, respectively; F simply waits passively for his payoff at the cash out stage. So a contract $\left(t, s, I_{F}, I_{V}, T_{F}, T_{V}\right)$ defines a simultaneous move game between $\mathrm{E}$ and $\mathrm{V}$, whose payoff functions are $p(e, a)(1-s)(1-t) \theta-U(e)+T_{F}+T_{V}$ and $p(e, a) s(1-t) \theta-V(a)-I_{V}-T_{V}$, respectively. The reaction functions are then given by

$$
e(a)=\arg \max _{e \in[0,1]} p(e, a)(1-s)(1-t) \theta-U(e),
$$

\footnotetext{
${ }^{27}$ Notice that by not requiring $T_{F} \geq 0$ and $T_{V} \geq 0$ we allow for transfers between $\mathrm{F}$ and $\mathrm{V}$.
} 


$$
a(e)=\arg \max _{a \in[0,1]} p(e, a) s(1-t) \theta-V(a) .
$$

By differentiating the corresponding first order conditions (which by concavity are necessary and sufficient) it is immediate to check that $e^{\prime}(a) \geq 0$ and $a^{\prime}(e) \geq 0$, with strict inequality in the interior of their respective domains. Clearly, $p(e, 0)=$ $p(0, a)=0$ implies that $(e, a)=(0,0)$ is always a Nash equilibrium. Moreover, if $e^{\prime}(0)>\left[a^{\prime}(0)\right]^{-1}$ there will be at least one additional intersection between the two reaction functions. If $\theta$ is not too large, we will have $e(1)<1$ and $a(1)<1$, in which case any additional equilibrium will be interior. But one can show that if $(e, a)$ is an interior equilibrium then $e^{\prime}(a)<\left[a^{\prime}(e)\right]^{-1}$, so such an equilibrium will be unique. In what follows we assume that the game between $\mathrm{E}$ and $\mathrm{V}$ always has an interior Nash equilibrium, and we let $\operatorname{intNE}(s,(1-t) \theta)$ denote the (singleton) set of interior Nash equilibria.

An optimal contract for a given $\theta$ solves the following problem:

$$
\max _{\left(t, s, I_{F}, I_{V}, T_{F}, T_{V}\right)} p(e, a)(1-s)(1-t) \theta-U(e)+T_{F}+T_{V}
$$

subject to

$$
\begin{gathered}
(e, a) \in \operatorname{intNE}(s,(1-t) \theta), \\
p(e, a) t \theta \geq I_{F}+T_{F}, \\
p(e, a) s(1-t) \theta-V(a) \geq I_{V}+T_{V}, \\
T_{F}+T_{V} \geq 0 .
\end{gathered}
$$

In words, the optimal contract maximizes E's equilibrium payoff subject to F's and V's individual rationality constraints, (27) and (28), respectively, and E's wealth constraint (29).

Clearly (27) and (28) will be satisfied with equality. Adding them up, solving for $T_{F}+T_{V}$, substituting the resulting expression into (25) and (29), and using the fact that $I_{F}+I_{V}=I_{1}$, one gets the following equivalent problem:

$$
\max _{(t, s)} p(e, a) \theta-U(e)-V(a)-I_{1}
$$

subject to (26) and

$$
p(e, a)[s(1-t)+t] \theta-V(a) \geq I_{1} .
$$


Notice that this formulation does not explicitly refer to $I_{F}, I_{V}, T_{F}$, and $T_{V}$, although if (31) is satisfied then the individual rationality constraints (27) and (28) would hold for, say, $I_{F}=0, I_{V}=I_{1}, T_{F}=p(e, a) t \theta$, and $T_{V}=p(e, a) s(1-t) \theta-V(a)-I_{1}$. In what follows we save on notation by describing contracts with the pair $(t, s)$.

Proposition A1 In an optimal contract, the project is fully funded by $V$, i.e. $I_{F}=T_{F}=t=0$.

Proof Suppose on the contrary that a contract $\left(t^{0}, s^{0}\right)$ with $t^{0}>0$ is optimal, and consider an alternative contract $\left(t^{1}, s^{1}\right)=\left(0, s^{0}\left(1-t^{0}\right)+t^{0}\right)$. The proof has three parts. We first characterize the equilibrium of the efforts game under the alternative contract. Next we show that the alternative contract is also feasible. Finally we prove that it yields a higher payoff to $\mathrm{E}$, which is a contradiction.

Part 1. By construction $\left(1-s^{1}\right)\left(1-t^{1}\right)=\left(1-s^{0}\right)\left(1-t^{0}\right)$, so it follows from (23) that the E's reaction function does not change: $e^{1}(a)=e^{0}(a)$ for all $a \in[0,1]$. In addition, $s^{1}\left(1-t^{1}\right)-s^{0}\left(1-t^{0}\right)=t^{0}>0$ implies from (24) that V's reaction function moves upwards: $a^{1}(e) \geq a^{0}(e)$ for all $e \in[0,1]$, with strict inequality for $e \in(0,1]$ such that $a^{0}(e) \in(0,1)$. Now let $\left(e^{0}, a^{0}\right)$ and $\left(e^{1}, a^{1}\right)$ denote the equilibrium efforts associated with the initial and the alternative contract, respectively. Then, given the way in which $e^{0}(a)$ and $a^{0}(e)$ intersect at $\left(e^{0}, a^{0}\right)$, the upward shift in V's reaction function implies $\left(e^{1}, a^{1}\right) \gg\left(e^{0}, a^{0}\right)$.

Part 2. To check that (31) holds for $(e, a) \in \operatorname{intNE}\left(s^{1}, \theta\right)$, let $a^{2}$ be implicitly defined by the equation $p\left(e^{1}, a^{2}\right)=p\left(e^{0}, a^{0}\right)$. Notice that $e^{1}>e^{0}$ implies $a^{2}<a^{0}$. Hence we have

$$
\begin{aligned}
p\left(e^{1}, a^{1}\right) s^{1} \theta-V\left(a^{1}\right) & >p\left(e^{1}, a^{2}\right) s^{1} \theta-V\left(a^{2}\right)>p\left(e^{0}, a^{0}\right) s^{1} \theta-V\left(a^{0}\right) \\
& =p\left(e^{0}, a^{0}\right)\left[s^{0}\left(1-t^{0}\right)+t^{0}\right] \theta-V\left(a^{0}\right) \geq I_{1}
\end{aligned}
$$

where the first inequality follows from the fact that $a^{1}=a^{1}\left(e^{1}\right)$, the second from $p\left(e^{1}, a^{2}\right)=p\left(e^{0}, a^{0}\right)$ and $V\left(a^{2}\right)<V\left(a^{0}\right)$, and the last from the feasibility of the initial contract.

Part 3. By (27), (28), and (32) we have

$$
\begin{aligned}
T_{F}^{1}+T_{V}^{1} & =p\left(e^{1}, a^{1}\right) s^{1} \theta-V\left(a^{1}\right)-I_{1} \\
& >p\left(e^{0}, a^{0}\right)\left[s^{0}\left(1-t^{0}\right)+t^{0}\right] \theta-V\left(a^{0}\right)-I_{1}=T_{F}^{0}+T_{V}^{0} .
\end{aligned}
$$


On the other hand, we have

$$
\begin{aligned}
p\left(e^{1}, a^{1}\right)\left(1-s^{1}\right) \theta-U\left(e^{1}\right) & >p\left(e^{0}, a^{1}\right)\left(1-s^{1}\right) \theta-U\left(e^{0}\right) \\
& >p\left(e^{0}, a^{0}\right)\left(1-s^{0}\right)\left(1-t^{0}\right) \theta-U\left(e^{0}\right)
\end{aligned}
$$

where the first inequality follows from the fact that $e^{1}=e^{1}\left(a^{1}\right)$, and the second from $a^{1}>a^{0}$. Hence we conclude that

$p\left(e^{1}, a^{1}\right)\left(1-s^{1}\right) \theta-U\left(e^{1}\right)+T_{F}^{1}+T_{V}^{1}>p\left(e^{0}, a^{0}\right)\left(1-s^{0}\right)\left(1-t^{0}\right) \theta-U\left(e^{0}\right)+T_{F}^{0}+T_{V}^{0}$,

which is a contradiction that establishes the result.

\section{B Proofs}

Proof of Lemma 1 Differentiating the payoff functions of $\mathrm{E}$ and V with respect to $e$ and $a$, respectively, solving the resulting first order conditions for these two variables, and taking into account their upper bound gives the following reaction functions:

$$
\begin{gathered}
e(a)=\min \left\{\left[u(1-\alpha)(1-s) \theta a^{\alpha}\right]^{\frac{1}{1+\alpha}}, 1\right\}, \\
a(e)=\min \left\{\left[v \alpha s \theta e^{1-\alpha}\right]^{\frac{1}{2-\alpha}}, 1\right\} .
\end{gathered}
$$

Since $e^{\prime}(0)=a^{\prime}(0)=+\infty$ these functions will have at least one intersection in addition to the one at the origin. Moreover since they are concave this intersection will be unique, but possibly with $e=1$ or $a=1$. To rule out these cases it suffices to ensure that $e(1)<1$ and $a(1)<1$. By $(33) e(1)<1$ if and only if $u(1-\alpha)(1-s) \theta<1$, that is if and only if $\theta<[u(1-\alpha)(1-s)]^{-1}$, and similarly by (34) $a(1)<1$ if and only if $\theta<[v \alpha s]^{-1}$. From here it follows that a sufficient condition for an interior equilibrium is

$$
\theta \leq \min \left\{[u(1-\alpha)]^{-1},[v \alpha]^{-1}\right\} .
$$

To compute this equilibrium we solve the system of equations

$$
e=\left[u(1-\alpha)(1-s) \theta a^{\alpha}\right]^{\frac{1}{1+\alpha}} \quad \text { and } \quad a=\left[v \alpha s \theta e^{1-\alpha}\right]^{\frac{1}{2-\alpha}}
$$

to get $e=[u(1-\alpha)(1-s) \rho(s)]^{\frac{1}{2}} \theta$ and $a=[v \alpha s \rho(s)]^{\frac{1}{2}} \theta$. Substituting these expressions into the function $p(e, a)$ gives (1), and into the payoff functions of $\mathrm{E}$ and $\mathrm{V}$ gives (2) and $(3)$. 
Proof of Lemma 2 By (4) we have $\rho(0)=\rho(1)=0$, which implies (10). To prove quasiconcavity we first show that $\ln \rho(s)$ is concave:

$$
\begin{gathered}
\frac{d \ln \rho(s)}{d s}=\frac{\alpha-s}{s(1-s)}, \\
\frac{d^{2} \ln \rho(s)}{d s^{2}}=-\frac{\alpha(1-s)^{2}+(1-\alpha) s^{2}}{s^{2}(1-s)^{2}}<0 .
\end{gathered}
$$

Next observe that

$$
\begin{gathered}
\frac{\partial \ln \left[\Pi(s, \theta)+I_{1}\right]}{\partial s}=\frac{1-2 \alpha}{1+\alpha+s-2 \alpha s}+\frac{d \ln \rho(s)}{d s}=\frac{\phi(s)}{s(1-s)(1+\alpha+s-2 \alpha s)}, \\
\frac{\partial^{2} \ln \left[\Pi(s, \theta)+I_{1}\right]}{\partial s^{2}}=-\left[\frac{1-2 \alpha}{1+\alpha+s-2 \alpha s}\right]^{2}+\frac{d^{2} \ln \rho(s)}{d s^{2}}<0 \\
\frac{\partial \ln \left[\Pi_{V}(s, \theta)+I_{1}\right]}{\partial s}=\frac{1}{s}+\frac{d \ln \rho(s)}{d s}=\frac{1+\alpha-2 s}{s(1-s)}, \\
\frac{\partial^{2} \ln \left[\Pi_{V}(s, \theta)+I_{1}\right]}{\partial s^{2}}=-\frac{1}{s^{2}}+\frac{d^{2} \ln \rho(s)}{d s^{2}}<0
\end{gathered}
$$

where

$$
\phi(s)=2(2 \alpha-1) s^{2}-2 \alpha(1+\alpha) s+\alpha(1+\alpha) .
$$

Thus, $\Pi(s, \theta)$ and $\Pi_{V}(s, \theta)$ are also quasiconcave in $s$. Moreover, it is clear that $\Pi_{V}(s, \theta)$ is maximized for $\widehat{s}=\frac{1}{2}(1+\alpha)$. On the other hand, for $\alpha=\frac{1}{2}$ the function $\phi(s)$ is linear and the solution to the equation $\phi\left(s^{*}\right)=0$ is $s^{*}=\frac{1}{2}$. For $0<\alpha<\frac{1}{2}$ the function $\phi(s)$ is quadratic with $\phi(\alpha)>0$ and $\phi\left(\frac{1}{2}\right)<0$, so there exists a unique $s^{*} \in\left(\alpha, \frac{1}{2}\right)$ for which $\phi\left(s^{*}\right)=0$. Finally, for $\frac{1}{2}<\alpha<1$ the function $\phi(s)$ is quadratic with $\phi\left(\frac{1}{2}\right)>0$ and $\phi(\alpha)<0$, so there exists a unique $s^{*} \in\left(\frac{1}{2}, \alpha\right)$ for which $\phi\left(s^{*}\right)=0$.

Proof of Proposition 1 It suffices to observe that by the definition of the function $\Pi_{V}(s, \theta)$ in Lemma 1 we have $\Pi_{V}(\widehat{s}, \theta) \geq 0$ if and only if

$$
\theta \geq \widehat{\theta}=\left[\frac{2 I_{1}}{(2-\alpha) \widehat{s} \rho(\widehat{s})}\right]^{\frac{1}{2}},
$$

and $\Pi_{V}\left(s^{*}, \theta\right) \geq 0$ if and only if

$$
\theta \geq \theta^{*}=\left[\frac{2 I_{1}}{(2-\alpha) s^{*} \rho\left(s^{*}\right)}\right]^{\frac{1}{2}} .
$$

Since $\widehat{s}$ maximizes $\Pi_{V}(s, \theta)$ and $\widehat{s} \neq s^{*}$ by Lemma 2 we have $\widehat{s} \rho(\widehat{s})>s^{*} \rho\left(s^{*}\right)$, which implies $\widehat{\theta}<\theta^{*}$. 
Proof of Proposition 2 By Lemma 2 we have $\max _{s} \Pi_{V}(s, \theta)=\Pi_{V}(\widehat{s}, \theta)$ and, by the definition of $\widehat{\theta}$ in the proof of Proposition 1 , we have $\Pi_{V}(\widehat{s}, \theta) \geq 0$ if and only if $\theta \geq \widehat{\theta}$. Hence we can define

$$
H=\max _{(\bar{\theta}, z(\theta))} \int_{\bar{\theta}}^{\infty} \Pi_{V}(z(\theta), \theta) d F(\theta)=\int_{\widehat{\theta}}^{\infty} \Pi_{V}(\widehat{s}, \theta) d F(\theta) .
$$

Clearly if $I_{0}>H$ no contract can satisfy V's individual rationality constraint (15), so the project is not feasible.

On the other hand, when $I_{0} \leq H$ there is a solution to the maximization of (14) subject to (15) that is characterized by the first order conditions:

$$
\begin{gathered}
\frac{\partial \Pi(z(\theta), \theta)}{\partial s}+\gamma \frac{\partial \Pi_{V}(z(\theta), \theta)}{\partial s}=0, \quad \text { for all } \theta \geq \bar{\theta} \\
\Pi(z(\bar{\theta}), \bar{\theta})+\gamma \Pi_{V}(z(\bar{\theta}), \bar{\theta})=0,
\end{gathered}
$$

where $\gamma \geq 0$ is the Lagrange multiplier of the constraint (15).

If this constraint is not binding, we have $\gamma=0$, in which case (36) implies $\partial \Pi(z(\theta), \theta) / \partial s=0$, so by the definition of $s^{*}$ in Lemma 2 we have $z(\theta)=s^{*}$ for all $\theta \geq \bar{\theta}$. Hence by (37) the cutoff point $\bar{\theta}$ satisfies $\Pi\left(s^{*}, \bar{\theta}\right)=0$. But since $\Pi\left(s^{*}, \widehat{\theta}\right)>\Pi(\widehat{s}, \widehat{\theta})>\Pi_{V}(\widehat{s}, \widehat{\theta})=0$, we conclude that $\bar{\theta}<\widehat{\theta}$. The compatibility of this unconstrained contract with (15) requires an initial investment $I_{0} \leq L$, where

$$
L=\int_{\bar{\theta}}^{\infty} \Pi_{V}\left(s^{*}, \theta\right) d F(\theta) .
$$

Clearly, we have $L<H$, and the transfer to $\mathrm{E}$ is simply $T=L-I_{0}$.

When $L<I_{0} \leq H$ the constraint (15) is binding and $\gamma>0$. In this case (36) implies that $z(\theta)$ solves

$$
-\frac{\partial \Pi(z(\theta), \theta)}{\partial s}\left(\frac{\partial \Pi_{V}(z(\theta), \theta)}{\partial s}\right)^{-1}=\gamma .
$$

But by (3) and (5) the LHS of this equation does not depend on $\theta$, so the solution involves a constant share $z(\theta)=\bar{s}$. Moreover since $\partial \Pi(s, \theta) / \partial s<0$ if and only if $s>s^{*}$, and $\partial \Pi_{V}(s, \theta) / \partial s>0$ if and only if $s<\widehat{s}$, we must have $\bar{s} \in\left(s^{*}, \hat{s}\right]$, with $\bar{s}=\widehat{s}$ in the limit case where $I_{0}=H$. To prove that the cutoff point $\bar{\theta}$ satisfies $\bar{\theta} \leq \widehat{\theta}$, notice that, otherwise, the proposed contract would be dominated by an alternative contract offering $\bar{s}$ for $\theta \geq \bar{\theta}$ and $\widehat{s}$ for a small set of states below $\bar{\theta}$. The alternative contract would increase the objective function $(14)$ by $\Pi(\widehat{s}, \bar{\theta}) F^{\prime}(\bar{\theta})$, and would relax the constraint $(15)$ by $\Pi_{V}(\widehat{s}, \bar{\theta}) F^{\prime}(\bar{\theta})$. But since $\Pi(\widehat{s}, \bar{\theta})>\Pi_{V}(\widehat{s}, \bar{\theta})>\Pi_{V}(\widehat{s}, \widehat{\theta})=0$, we have a contradiction. 
Proof of Proposition 3 By Lemma 2 we have $\max _{s} \Pi_{V}(s, \theta)=\Pi_{V}(\widehat{s}, \theta)$ and, by the definition of $\widehat{\theta}$ in the proof of Proposition 1 , we have $\Pi_{V}(\widehat{s}, \theta) \geq 0$ if and only if $\theta \geq \widehat{\theta}$. Hence V's compensation is maximized by setting $C(\theta)=\Pi_{V}(\widehat{s}, \theta)$ for all $\theta \geq \widehat{\theta}$, in which case by the definition of $H$ in (35) we have $\int_{\widehat{\theta}}^{\infty} C(\theta) d F(\theta)=H$. Clearly, if $I_{0}>H$, V's individual rationality constraint (18) cannot be satisfied.

On the other hand, when $I_{0} \leq H$ the optimal compensation problem has a solution. The Lagrangian corresponding to this problem is:

$$
\left.\int_{\widehat{\theta}}^{\infty} \Pi(s(\theta, C(\theta)), \theta)+\lambda C(\theta)+\mu(\theta) C(\theta)\right] d F(\theta)-(1+\lambda) I_{0}
$$

where $\lambda \geq 0$ and $\mu(\theta) \geq 0$ are the Lagrange multipliers of the constraints (18) and (19), respectively. Differentiating (39) with respect to $C(\theta)$ gives the first order condition

$$
\frac{\partial \Pi(s(\theta, C(\theta)), \theta)}{\partial s} \frac{\partial s(\theta, C(\theta))}{\partial C}+\lambda+\mu(\theta)=0, \quad \text { for all } \theta \geq \widehat{\theta}
$$

When V's individual rationality constraint (18) is not binding, we have $\lambda=0$. Now if the nonnegativity constraint $C(\theta) \geq 0$ is also not binding, we have $\mu(\theta)=0$, in which case (40) implies $\partial \Pi(s(\theta, C(\theta)), \theta) / \partial s=0$, so by the definition of $s^{*}$ in Lemma 2 we have $s(\theta, C(\theta))=s^{*}$. Hence by the definition (16) of $s(\theta, C)$ it must be the case that $C(\theta) \leq \Pi_{V}\left(s^{*}, \theta\right)$. But by Proposition 1 we have $\Pi_{V}\left(s^{*}, \theta\right)<0$ for $\theta<\theta^{*}$, so for $\theta \in\left[\widehat{\theta}, \theta^{*}\right)$ we must have $C(\theta)=0$ and $s(\theta, C(\theta))=s(\theta)$. Clearly, this solution obtains when

$$
I_{0} \leq L^{\prime}=\int_{\theta^{*}}^{\infty} \Pi_{V}\left(s^{*}, \theta\right) d F(\theta) .
$$

Since $\Pi_{V}(\widehat{s}, \theta)>\Pi_{V}\left(s^{*}, \theta\right)>0$ for $\theta>\theta^{*}$ and $\Pi_{V}(\widehat{s}, \theta) \geq 0$ for $\widehat{\theta} \leq \theta \leq \theta^{*}$, comparing (35) with (41) we get $L^{\prime}<H$.

When $L^{\prime}<I_{0} \leq H$ the constraint (18) is binding and $\lambda>0$. This implies that the first term in the LHS of the first order condition (40) must be negative, which implies $\partial s(\theta, C(\theta)) / \partial C>0$. But then by (16) it must be the case that

$$
\Pi_{V}(s(\theta, C(\theta)), \theta)=C(\theta)
$$

so

$$
\frac{\partial \Pi_{V}(s(\theta, C(\theta)), \theta)}{\partial s} \frac{\partial s(\theta, C(\theta))}{\partial C}=1
$$


Substituting this expression into (40) yields

$$
-\frac{\partial \Pi(s(\theta, C(\theta)), \theta)}{\partial s}\left(\frac{\partial \Pi_{V}(s(\theta, C(\theta)), \theta)}{\partial s}\right)^{-1}=\lambda+\mu(\theta) .
$$

By (3) and (5) the LHS of this equation does not depend on $\theta$. Hence, when the constraint $C(\theta) \geq 0$ is not binding, $\mu(\theta)=0$ implies that the induced expansion stage contract does not depend on $\theta$, that is $\widetilde{s}(\theta)=s(\theta, C(\theta))=\widetilde{s}$ for some $\widetilde{s} \in\left[\widehat{s}, s^{*}\right)$, in which case $C(\theta)=\Pi_{V}(\widetilde{s}, \theta)$. On the other hand, when $C(\theta) \geq 0$ is binding, we have $C(\theta)=0$ and $\widetilde{s}(\theta)=s(\theta, 0)=s(\theta)$. Finally, it is clear that the critical value $\widetilde{\theta} \in\left(\widehat{\theta}, \theta^{*}\right)$ below which the constraint $C(\theta) \geq 0$ is binding is implicitly defined by the condition $\Pi_{V}(\widetilde{s}, \widetilde{\theta})=0$, so $\widetilde{s}=s(\widetilde{\theta})$.

Proof of Proposition 4 For $\widehat{\theta} \leq \theta \leq \widetilde{\theta}$ Proposition 3 establishes that $C(\theta)=0$ and $s(\theta, 0)=s(\theta)$. But then (21) holds if and only if $\theta^{\prime}=\theta$, which implies $r(\theta)=0$.

For $\theta>\tilde{\theta}$ Proposition 3 establishes that $C(\theta)=\Pi_{V}(\widetilde{s}, \theta)$ and $s(\theta, C(\theta))=\widetilde{s}$. Hence (21) becomes

$$
\Pi\left(s\left(\theta^{\prime}\right), \theta^{\prime}\right)=\Pi(\widetilde{s}, \theta)-\Pi_{V}(\widetilde{s}, \theta) .
$$

To prove that this equation has a unique solution, notice that for $r=0$ we have $\theta^{\prime}=(1-r(\theta)) \theta=\theta>\widetilde{\theta}$, so $s\left(\theta^{\prime}\right)=s(\theta)<s(\widetilde{\theta})=\widetilde{s}$ implies

$$
\left.\Pi\left(s\left(\theta^{\prime}\right), \theta^{\prime}\right)\right|_{r=0}=\Pi(s(\theta), \theta)>\Pi(\widetilde{s}, \theta)>\Pi(\widetilde{s}, \theta)-\Pi_{V}(\widetilde{s}, \theta),
$$

where we have used that $\Pi_{V}(\widetilde{s}, \theta)>0$. On the other hand for $r=1-\widetilde{\theta} / \theta$ we have $\theta^{\prime}=(1-r(\theta)) \theta=\widetilde{\theta}<\theta$, so $s\left(\theta^{\prime}\right)=s(\widetilde{\theta})=\widetilde{s}$ imply

$$
\left.\Pi\left(s\left(\theta^{\prime}\right), \theta^{\prime}\right)\right|_{r=1-\widetilde{\theta} / \theta}=\Pi(\widetilde{s}, \widetilde{\theta})<\Pi(\widetilde{s}, \theta)-\Pi_{V}(\widetilde{s}, \theta),
$$

where we have used that $\Pi(\widetilde{s}, \widetilde{\theta})=\Pi_{E}(\widetilde{s}, \widetilde{\theta})$ (since $\Pi_{V}(\widetilde{s}, \widetilde{\theta})=0$ ) and $\Pi_{E}(\widetilde{s}, \widetilde{\theta})<$ $\Pi_{E}(\widetilde{s}, \theta)=\Pi(\widetilde{s}, \theta)-\Pi_{V}(\widetilde{s}, \theta)$. By continuity, these two results imply the existence of $r(\theta) \in(0,1-\tilde{\theta} / \theta)$ that satisfies (42). Moreover since $\Pi\left(s\left(\theta^{\prime}\right), \theta^{\prime}\right)$ is obviously decreasing in $r, r(\theta)$ is unique.

To prove that $r^{\prime}(\theta)>0$ for $\theta>\widetilde{\theta}$, consider first the case where $\theta^{\prime}<\theta^{*}$. In this case $s\left(\theta^{\prime}\right)>s^{*}$, so $\Pi_{V}\left(s\left(\theta^{\prime}\right), \theta^{\prime}\right)=0$ and we can express (42) as

$$
\Pi_{E}\left(s\left(\theta^{\prime}\right), \theta^{\prime}\right)=\Pi_{E}(\widetilde{s}, \theta) .
$$


Totally differentiating this equation with respect to $\theta$ gives

$$
\left(\frac{\partial \Pi_{E}\left(s\left(\theta^{\prime}\right), \theta^{\prime}\right)}{\partial s} \frac{d s\left(\theta^{\prime}\right)}{d \theta^{\prime}}+\frac{\partial \Pi_{E}\left(s\left(\theta^{\prime}\right), \theta^{\prime}\right)}{\partial \theta^{\prime}}\right)\left(1-r(\theta)-\theta r^{\prime}(\theta)\right)=\frac{\partial \Pi_{E}(\widetilde{s}, \theta)}{\partial \theta} .
$$

Using (2) and (43) we have

$$
\frac{\partial \Pi_{E}(\widetilde{s}, \theta)}{\partial \theta}=\frac{2 \Pi_{E}(\widetilde{s}, \theta)}{\theta}=\frac{2 \Pi_{E}\left(s\left(\theta^{\prime}\right), \theta^{\prime}\right)}{\theta^{\prime}}(1-r(\theta))=\frac{\partial \Pi_{E}\left(s\left(\theta^{\prime}\right), \theta^{\prime}\right)}{\partial \theta^{\prime}}(1-r(\theta)),
$$

so (44) gets reduced to

$$
\frac{\partial \Pi_{E}\left(s\left(\theta^{\prime}\right), \theta^{\prime}\right)}{\partial s} \frac{d s\left(\theta^{\prime}\right)}{d \theta^{\prime}}(1-r(\theta))=\left(\frac{\partial \Pi_{E}\left(s\left(\theta^{\prime}\right), \theta^{\prime}\right)}{\partial s} \frac{d s\left(\theta^{\prime}\right)}{d \theta^{\prime}}+\frac{\partial \Pi_{E}\left(s\left(\theta^{\prime}\right), \theta^{\prime}\right)}{\partial \theta^{\prime}}\right) \theta r^{\prime}(\theta) .
$$

But from the analysis in Section 3 we know that $\partial \Pi_{E} / \partial s<0, \partial \Pi_{E} / \partial \theta^{\prime}>0$, and $d s\left(\theta^{\prime}\right) / d \theta^{\prime}<0$, so we must have $r^{\prime}(\theta)>0$. Finally, consider the case where $\theta^{\prime} \geq \theta^{*}$. Now we have $s\left(\theta^{\prime}\right)=s^{*}$ so $(42)$ can be written as

$$
\Pi\left(s^{*}, \theta^{\prime}\right)=\Pi_{E}(\widetilde{s}, \theta) .
$$

Differentiating this equation with respect to $\theta$ gives

$$
\frac{\partial \Pi\left(s^{*}, \theta^{\prime}\right)}{\partial \theta^{\prime}}\left(1-r(\theta)-\theta r^{\prime}(\theta)\right)=\frac{\partial \Pi_{E}(\widetilde{s}, \theta)}{\partial \theta} .
$$

Using (2), (5), and (45) we have

$$
\frac{\partial \Pi_{E}(\widetilde{s}, \theta)}{\partial \theta}=\frac{2 \Pi_{E}(\widetilde{s}, \theta)}{\theta}=\frac{2 \Pi\left(s^{*}, \theta^{\prime}\right)}{\theta}=\frac{\partial \Pi\left(s^{*}, \theta^{\prime}\right)}{\partial \theta^{\prime}}(1-r(\theta))-\frac{2 I_{1}}{\theta},
$$

so (46) gets reduced to

$$
\frac{\partial \Pi\left(s^{*}, \theta^{\prime}\right)}{\partial \theta^{\prime}} \theta r^{\prime}(\theta)=\frac{2 I_{1}}{\theta}
$$

which implies $r^{\prime}(\theta)>0$ since $\partial \Pi / \partial \theta^{\prime}>0$. 


\section{References}

[1] Admati, A. R. and P. Pfleiderer (1994), "Robust Financial Contracting and the Role of Venture Capitalists," Journal of Finance, 49, 371-402.

[2] Bergemann, D. and U. Hege (1998), "Venture Capital Financing, Moral Hazard and Learning," Journal of Banking and Finance, 22, 703-735.

[3] Berglof, E. (1994), "A Control Theory of Venture Capital Finance," Journal of Law, Economics, and Organization, 10, 247-267.

[4] Casamatta, C. (2003), "Financing and Advising: Optimal Financial Contracts with Venture Capitalists," Journal of Finance, 58, 2059-2086.

[5] Cornelli, F. and O. Yosha (2003), "Stage Financing and the Role of Convertible Securities," Review of Economic Studies, 70, 1-32.

[6] Eswaran, M. and A. Kotwal (1984), "The Moral Hazard of Budget-Breaking," Rand Journal of Economics, 15, 578-581.

[7] Gompers, P. A. (1995), "Optimal Investment, Monitoring, and the Staging of Venture Capital," Journal of Finance, 50, 1461-1489.

[8] Gompers, P. A. (1997), "Ownership and Control in Entrepreneurial Firms: An Examination of Convertible Securities in Venture Capital Investments," unpublished manuscript, Harvard University.

[9] Gorman, M. and W. A. Sahlman (1989), "What Do Venture Capitalists Do?" Journal of Business Venturing, 5, 77-90.

[10] Hart, O. (2001), "Financial Contracting," Journal of Economic Literature, 39, 1079-1100.

[11] Hart, O. and J. Moore (1998), "Default and Renegotiation: A Dynamic Model of Debt," Quarterly Journal of Economics, 113, 1-41.

[12] Holmström, B. (1982), "Moral Hazard in Teams," Bell Journal of Economics, $13,324-340$. 
[13] Inderst, R. and H. M. Müller (2003), "The Effect of Capital Market Characteristics on the Value of Start-Up Firms," Journal of Financial Economics, forthcoming.

[14] Kaplan, S. N. and P. Strömberg (2003), "Financial Contracting Theory Meets the Real World: An Empirical Analysis of Venture Capital Contracts," Review of Economic Studies, 70, 281-315.

[15] Kaplan, S. N. and P. Strömberg (2001), "Venture Capitalists as Principals: Contracting, Screening, and Monitoring," American Economic Review Papers and Proceedings, 91, 426-430.

[16] Marx, L. M. (1998), "Efficient Venture Capital Financing Combining Debt and Equity," Review of Economic Design, 3, 371-387.

[17] Neher, D. V. (1999), "Staged Financing: An Agency Perspective," Review of Economic Studies, 66, 255-274.

[18] Osborne, M. J., and A. Rubinstein (1990), Bargaining and Markets, Academic Press, San Diego.

[19] Repullo, R. and J. Suarez, "Monitoring, Liquidation, and Security Design," Review of Financial Studies, 11, 163-187.

[20] Sahlman, W. A. (1990), "The Structure and Governance of Venture-Capital Organizations," Journal of Financial Economics, 27, 473-521.

[21] Schmidt, K. M. (2003), "Convertible Securities and Venture Capital Finance," Journal of Finance, 58, 1139-1166.

[22] Sutton, J. (1986), "Non-Cooperative Bargaining Theory: An Introduction," Review of Economic Studies, 53, 709-724.

[23] Trester, J. J. (1998), "Venture Capital Contracting under Asymmetric Information," Journal of Banking and Finance, 22, 675-699. 